Japan. J. Math.

Vol. 17, No.2, 1991

\title{
The orbital decomposition of a representation induced from a nil-subgroup
}

\author{
By Ronald L. Lipsman* \\ (Received December 1990)
}

\section{Introduction. Philosophy and statement of main results}

Let $G$ be a simply connected solvable type I Lie group. One knows how to parameterize the irreducible unitary representations of $G$ [1]. Namely, if $\mathfrak{g}=L A(G)$ and $\mathfrak{g}^{*}$ is its real dual, then $\widehat{G}$ is a "fiber space" with the set of co-adjoint orbits $\mathfrak{g}^{*} / G$ as base, and fiber

$$
\mathcal{X}(\varphi)=\left\{\tau \in \widehat{G}_{\varphi}: d \tau=d \chi_{\varphi}\right\}
$$

Here $\varphi \in \mathfrak{g}^{*}, G_{\varphi}$ is its stability group and $\chi_{\varphi}$ is the unique unitary character of the identity component $G_{\varphi}^{0}$ satisfying $d \chi_{\varphi}=\left.i \varphi\right|_{\mathfrak{g}_{\varphi}}$. The structure groups $G_{\varphi} / G_{\varphi}^{0}$ are free abelian on finitely many generators [1], so the fibers $\mathcal{X}(\varphi)$ are tori. The main problem we are concerned with here is the following. If $H \subset G$ is a closed connected (therefore simply connected) subgroup of $G$ and $\nu \in \widehat{H}$, we would like to give a direct integral decomposition of the induced representation $\operatorname{Ind}_{H}^{G} \nu$ in terms of the orbital parameters of $\widehat{G}$ described above. The problem has been solved when $G$ is nilpotent [3] [7], and more generally when $G$ is completely solvable [8] or exponential solvable [4] [9]. In those cases the fibers $\mathcal{X}(\varphi)$ are singletons and the solution is: if $\nu=\nu_{\psi} \in \widehat{H}, \psi \in \mathfrak{h}^{*}$, then

$$
\operatorname{Ind}_{H}^{G} \nu_{\psi}=\int_{p^{-1}(H \cdot \psi) / H}^{\oplus} \pi_{\varphi} d \dot{\varphi}=\int_{G^{-1}(H \cdot \psi) / G}^{\oplus} n_{\varphi}^{\psi} \pi_{\varphi} d \ddot{\varphi} .
$$

Here $p: \mathfrak{g}^{*} \rightarrow \mathfrak{h}^{*}$ is the canonical projection, the measures $d \dot{\varphi}, d \ddot{\varphi}$ are push-forwards of the canonical measure class on $p^{-1}(H \cdot \psi)$ - that being a fiber measure with canonical $H$-invariant measure on the base $H \cdot \psi$ and Lebesgue measure on the affine fiber $\mathfrak{h}^{\perp}$ - and

$$
n_{\varphi}^{\psi}=\#\left(G \cdot \varphi \cap p^{-1}(H \cdot \psi)\right) / H
$$

*Research supported by NSF \#90-02642. 
The first direct integral in formula (1.1) has irreducible representations as constituents, but the equivalence class of representations corresponding to different $H$-orbits may be the same. This is because two points within the same $G$-orbit may be in distinct $H$-orbits. That is accounted for in the second direct integral where the exact multiplicity is computed.

The orbit method philosophy and the weight of evidence suggests a natural generalization of the decomposition (1.1) to non-exponential solvable Lie groups. Assume that both $G$ and $H$ are simply connected solvable and type I. Let $\nu=$ $\nu_{\psi, \sigma} \in \widehat{H}$ where $\psi \in \mathfrak{h}^{*}, \sigma \in \mathcal{X}(\psi)$. Then one may conjecture that

$$
\operatorname{Ind}_{H}^{G} \nu_{\psi, \sigma}=\int_{p^{-1}(H \cdot \psi) / H}^{\oplus} \int_{\mathcal{X}_{\sigma}(\varphi)}^{\oplus} \pi_{\varphi, \tau} d \tau d \dot{\varphi}
$$

where $\mathcal{X}_{\sigma}(\varphi)=\left\{\tau \in \mathcal{X}(\varphi):\left.\tau\right|_{H_{\varphi}}=\left.\sigma\right|_{H_{\varphi}}\right\}, d \tau$ is Lebesgue measure, and $d \dot{\varphi}$ is as in (1.1). There are several difficulties with formula (1.3) that must be confronted immediately. First of all, the set $\mathcal{X}_{\sigma}(\varphi)$ can be empty (for some $\varphi \in p^{-1}(H \cdot \psi)$ ). A necessary condition for $\tau \in \mathcal{X}(\varphi)$ to satisfy $\left.\tau\right|_{H_{\varphi}}=\left.\sigma\right|_{H_{\varphi}}$ is that $\chi_{\varphi}$ and $\sigma$ agree on $G_{\varphi}^{0} \cap H_{\varphi}$. But that intersection can be larger than $H_{\varphi}^{0}$. A less serious difficulty is that, even if non-empty, the set $\mathcal{X}_{\sigma}(\varphi)$ might only be a disconnected union of tori. (These problems are addressed to some extent in [10. Theorem 2].) A more serious problem is that one cannot pass - as we did in formula (1.1) - to a second explicit multiplicity formula. This is because when $\varphi_{1}, \varphi_{2}$ lie in the same $G$-orbit - forcing $G_{\varphi_{1}}$ and $G_{\varphi_{2}}$ to be conjugate - it does not follow that $H_{\varphi_{1}}$ and $H_{\varphi_{2}}$ are conjugate. This makes it difficult to count the multiplicity. Such difficulties disappear when $H$ is normal (obviously); but also when $H$ acts nilpotently on $G$ (see the discussion below). Finally a much more serious problem is the fact that formula (1.3) can actually be false. An example is given in [7, Example 8. vii] wherein $G$ is an unusual 6 -dimensional oscillator group and $H$ is a maximal torus. (The groups are not simply connected, but the same phenomenon persists for the simply connected coverings.) Nevertheless formula (1.3) is true for many homogeneous spaces - even non-solvable homogeneous spaces (see $[7, \S 8]$ ). Thus it is of interest to discover criteria under which formula (1.3) actually holds. The main purpose of this paper is to give a sufficient condition.

The condition originated in an attempt to understand how the proof of (1.1) in the nilpotent or exponential solvable case might break down in general. The method of proof in [7], [8] or [9] is via induction on $\operatorname{dim} G / H$. The linchpin of the argument is our ability to reduce to and handle the co-dimension 1 situation in [7] and [8], and the co-dimension 2 situation in [9]. It is the latter wherein the breakdown occurs - the passage from spiral-like orbits to circular orbits can foul up the works. But it appears to me that no anomalies can arise in the co-dimension 1 situation. Thus I have formulated the following. 
Working Conjecture. Suppose there is a sequence of closed simply connected type I subgroups $H=G_{0} \subset G_{1} \subset \cdots \subset G_{n-1} \subset G_{n}=G$ satisfying $\operatorname{dim} G_{i} / G_{i-1}=$ $1,1 \leq i \leq n$. Then formula (1.3) is valid.

The main result of the paper is the verification of the Working Conjecture under the imposition of two additional hypotheses. The first hypothesis is designed to deal with the conjugacy problem described in the paragraph above (following (1.3)). As indicated there, that problem can be overcome by assuming $H$ is normal - an unreasonably strong assumption - or $H$ acts nilpotently. We shall assume $H \subset$ Nilrad $G$. This also overcomes the non-emptiness difficulty described in the same paragraph above. The second assumption conforms to the step in the proof of formula (1.1) for completely solvable groups wherein we handle nilpotent groups first. Namely we shall assume that each $G_{i-1}$ is normal in $G_{i}$. This greatly simplifies the induction argument. Actually, it is in some sense a redundant assumption when $H \subset$ Nilrad $G$. For in that case one can always find a tower of co-dimension 1 normal subgroups. However conceivably some of the groups in the tower could be non-type I. Thus, although natural, the assumption is not redundant. Indeed our proof of the Working Conjecture will require $H \subset$ Nilrad $G$ and $G_{i-1} \triangleleft G_{i}$, but I believe it is true without these assumptions and $I$ hope to return to that issue at a later time.

Now I give precise statements of the two main results of the paper. The first corresponds to the Working Conjecture at the level $G_{i-1} \triangleleft G_{i}$. The second is the conjecture itself in the greatest generality provable at this stage.

Theorem 1. Let $N \triangleleft G$ be simply connected solvable type I Lie groups with $\operatorname{dim} G / N=1$. Let $\gamma=\gamma_{\theta, \sigma} \in \widehat{N}, \theta \in \mathfrak{n}^{*}, \sigma \in \mathcal{X}(\theta)$. There are four possibilities for the structure of the induced representation $\pi=\operatorname{Ind}_{N}^{G} \gamma_{\theta, \sigma}$ according as: (i) $G_{\theta}=N_{\theta}$; (ii) $G_{\theta} / N_{\theta} \cong \mathbf{Z}$; (iii) $G_{\theta} / N_{\theta} \cong \mathbf{R}$ and $G_{\theta}=\left(G_{\theta}\right)_{\sigma}$; (iv) $G_{\theta} / N_{\theta} \cong \mathbf{R}$ and $G_{\theta} \neq\left(G_{\theta}\right)_{\sigma}$. These structures are specified as follows:

(i) There is a unique $G$-orbit $G \cdot \varphi$ containing $p^{-1}(N \cdot \theta)$ and $\mathcal{X}_{\sigma}(\varphi)$ is a singleton $\{\tau\}$. The representation $\pi$ is irreducible and equivalent to $\pi_{\varphi, \tau}$.

(ii) There is a unique $G$-orbit $G \cdot \varphi$ containing $p^{-1}(N \cdot \theta)$, but $\mathcal{X}_{\sigma}(\varphi)$ is a 1 -torus. Then $\pi$ is a direct integral of irreducibles $\pi=\int_{\mathcal{X}_{\sigma}(\varphi)}^{\oplus} \pi_{\varphi, \tau} d \tau$.

(iii) There is a one-parameter family $G \cdot \varphi_{t}, t \in \mathbf{R}$, of distinct $G$-orbits meeting $p^{-1}(N \cdot \theta)$, and $\mathcal{X}_{\sigma}\left(\varphi_{t}\right)$ is a singleton $\left\{\tau_{t}\right\}$ for each $t \in \mathbf{R}$. Then $\pi$ is a direct integral of irreducibles $\pi=\int_{\mathbf{R}}^{\oplus} \pi_{\varphi_{t}, \tau_{t}} d t$.

(iv) There is a one-parameter family $G \cdot \varphi_{t}, t \in \mathbf{R}$, of $G$-orbits meeting $p^{-1}(N \cdot \theta)$, however $\exists t_{0} \neq 0$ such that $G \cdot \varphi_{t}=G \cdot \varphi_{t^{\prime}} \Leftrightarrow t \equiv t^{\prime} \bmod t_{0}$. The fibers $\mathcal{X}_{\sigma}\left(\varphi_{t}\right)$ are again singletons $\left\{\tau_{t}\right\}$. Then $\pi$ is a direct integral of irreducibles $\pi=\int_{\left[0, t_{0}\right]}^{\oplus} \pi_{\varphi_{t}, \tau_{t}} d t$

In all four cases formula (1.3) is satisfied, that is $\operatorname{Ind}_{N}^{G} \gamma_{\theta, \sigma}=\int_{p^{-1}(N \cdot \theta) / N}^{\oplus}$ 
$\int_{\mathcal{X}_{\sigma}(\varphi)}^{\oplus} \pi_{\varphi, \tau} d \tau d \dot{\varphi}$. In every instance the decomposition is multiplicity-free so that we also have $\operatorname{Ind}_{N}^{G} \gamma_{\theta, \sigma}=\int_{G \cdot p^{-1}(N \cdot \theta) / G}^{\oplus} \int_{\mathcal{X}_{\sigma}(\varphi)}^{\oplus} \pi_{\varphi, \tau} d \tau d \ddot{\varphi}$.

Theorem 2. Let $H \subset G$, where $G$ is simply connected solvable type I and $H$ is closed, connected and $\subset$ Nilrad $G$. Assume there is a sequence of closed connected type I subgroups $H=G_{0} \subset G_{1} \subset \cdots \subset G_{n-1} \subset G_{n}=G$ such that $G_{i-1} \triangleleft G_{i}$ and $\operatorname{dim} G_{i} / G_{i-1}=1,1 \leq i \leq n$. Then for any $\nu=\nu_{\psi} \in \widehat{H}$, we have

$$
\begin{aligned}
\operatorname{Ind}_{H}^{G} \nu_{\psi} & =\int_{p^{-1}(H \cdot \psi) / H}^{\oplus} \int_{\mathcal{X}(\varphi)}^{\oplus} \pi_{\varphi, \tau} d \tau d \dot{\varphi} \\
& =\int_{G \cdot p^{-1}(H \cdot \psi) / G}^{\oplus} n_{\varphi}^{\psi} \int_{\mathcal{X}(\varphi)}^{\oplus} \pi_{\varphi, \tau} d \tau d \ddot{\varphi} .
\end{aligned}
$$

We shall prove Theorem 1 in Section 2 by a structure theory argument. Not unexpectedly, this requires keeping very close track of both Mackey and orbital parameters as well as the relations between them. In Section 3 we utilize Theorem 1 in order to construct an induction argument for the proof of Theorem 2. As is usual in this kind of theorem, we shall encounter a dichotomy according to whether $H$ has open orbits on the intersection $G \cdot \varphi \cap p^{-1}(H \cdot \psi)$ or not.

We close this introduction with a remark and two points of notation. The remark is that one can formulate an analog to the Working Conjecture for restricted representations - namely, under the same assumptions, one has

$$
\left.\pi_{\varphi, \tau}\right|_{H}=\int_{p(G \cdot \varphi) / H}^{\oplus} n_{\varphi}^{\psi} \int_{\mathcal{X}_{+}(\psi)}^{\oplus} \nu_{\psi, \sigma} d \sigma d \dot{\psi} .
$$

I can prove analogs to Theorems 1 and 2 , but I leave that to another time. The point of notation is that if $p: \mathfrak{g}^{*} \rightarrow \mathfrak{h}^{*}$ is the canonical projection, we write $p=p_{\mathfrak{g}, \mathfrak{h}}$ if we need to specify the algebras. Similarly we write $\mathfrak{h}^{\perp}(\mathfrak{g})=\left\{\varphi \in \mathfrak{g}^{*}: \varphi(\mathfrak{h})=0\right\}$ if we need to specify the superalgebra in which the perp is taken.

\section{Co-dimension one}

We prove Theorem 1 now. Let $N \triangleleft G$ be simply connected solvable type I Lie groups with $\operatorname{dim} G / N=1$. Let $\theta \in \mathfrak{n}^{*}, \sigma \in \mathcal{X}(\theta)$ and $\gamma=\gamma_{\theta, \sigma} \in \widehat{N}$. We write $N_{\theta}^{0}$ for the identity component of $N_{\theta}$ and $\chi_{\theta}$ for the unitary character of $N_{\theta}^{0}$ satisfying $\chi_{\theta}(\exp X)=e^{i \theta(X)}, X \in \mathfrak{n}_{\theta}$ (alternatively $\left.d \chi_{\theta}=\left.i \theta\right|_{\mathfrak{n}_{\theta}}\right)$. Since $N_{\theta} \triangleleft G_{\theta}, G_{\theta}$ acts on $\mathcal{X}(\theta)$; we write $\left(G_{\theta}\right)_{\sigma}$ for the stability group of $\sigma \in \mathcal{X}(\theta)$. Then the stability group in $G$ of $\gamma=\gamma_{\theta, \sigma}$ is clearly

$$
G_{\gamma}=\left(G_{\theta}\right)_{\sigma} N
$$

We quickly recall the Mackey theory (see [5]) for the irreducible representations of $G$ which lie over $\gamma$. Since $G / N \cong \mathbf{R}$, the group $G_{\gamma} / N$ is either trivial, $\mathbf{R}$, or finite 
abelian on 1 generator. In any of these instances there is no possible non-trivial Mackey obstruction, so $\gamma$ extends to an ordinary (non-projective) representation $\widetilde{\gamma}$ of $G_{\gamma}$. The irreducibles of $G$ lying over $\gamma$ are then $\pi_{\gamma, \rho}=\operatorname{Ind}_{G_{\gamma}}^{G} \rho \tilde{\gamma}$, where $\rho$ is any character of $G_{\gamma}$ trivial on $N, \rho \in\left(G_{\gamma} / N\right)^{\wedge}$. It is then a basic result of [5] that

$$
\operatorname{Ind}_{N}^{G} \gamma=\int_{\left(G_{\gamma} / N\right)^{\wedge}}^{\oplus} \pi_{\gamma, \rho} d \rho, \quad d \rho=\text { Haar measure. }
$$

Next we choose an element $X \in \mathfrak{g}, X \notin \mathfrak{n}$, and define $\alpha \in \mathfrak{n}^{\perp}(\mathfrak{g})$ by letting $\alpha(X)=1$. We consider these choices fixed. We examine the four possibilities.

(i) $G_{\theta}=N_{\theta}$

(ii) $G_{\theta} / N_{\theta} \cong \mathbf{Z}$

(iii) $G_{\theta} / N_{\theta} \cong \mathbf{R}$ and $G_{\theta}=\left(G_{\theta}\right)_{\sigma}$

(iv) $G_{\theta} / N_{\theta} \cong \mathbf{R}$ and $G_{\theta} \neq\left(G_{\theta}\right)_{\sigma}$.

Since $G_{\theta} / N_{\theta}=G_{\theta} /\left(N \cap G_{\theta}\right) \cong G_{\theta} N / N$ is a closed subgroup of $G / N \cong \mathbf{R}$, the four possibilities are exhaustive. They are clearly mutually exclusive. That each occurs is illustrated by the first four examples at the end of the section. We examine the cases individually.

(i) $G_{\theta}=N_{\theta}$. Then clearly $G_{\gamma}=N$, and so $\pi=\operatorname{Ind}_{N}^{G} \gamma$ must be irreducible. Let $\varphi \in \mathfrak{g}^{*}$ be defined by $\left.\varphi\right|_{\mathfrak{n}}=\theta, \varphi(X)=0$. Then by a standard Lemma $[8$, Lemma 2.4] we have

$$
N_{\theta}^{0} \cdot \varphi=\varphi+\mathfrak{g}_{\gamma}^{\perp}=\varphi+\mathfrak{n}^{\perp}
$$

Hence there is only one $G$-orbit lying over $\theta$. Next we observe that

$$
G_{\varphi}=\left(G_{\theta}\right)_{\varphi}=\left(N_{\theta}\right)_{\varphi}=N_{\varphi} \subset N_{\theta}
$$

Set $\tau=\left.\sigma\right|_{G_{\varphi}}$. Then $\tau \in \mathcal{X}_{\sigma}(\varphi)$ and clearly $\mathcal{X}_{\sigma}(\varphi)=\{\tau\}$. It remains to prove that $\pi=\pi_{\varphi, \tau}$. That is accomplished using the fundamental Auslander-Kostant [1] construction of the irreducible representation corresponding to the data $(\varphi, \tau)$. Let $\mathfrak{u}_{\mathfrak{n}}$ denote the nilradical of $\mathfrak{n}$. One knows [1] that one can find a complex subalgebra $\left(\mathfrak{n}_{\theta}\right)_{c} \subset \mathfrak{b} \subset \mathfrak{n}_{c}$, which is a positive polarization for $\theta$, and with the additional properties that it is $N_{\theta}$-invariant, satisfies the Pukanszky condition, and is admissible with respect to $\mathfrak{u}_{\mathfrak{n}}$. (The latter means $\mathfrak{b} \cap\left(\mathfrak{u}_{\mathfrak{n}}\right)_{c}$ has the same properties with respect to $\left.\theta\right|_{\mathfrak{u}_{\mathfrak{n}}}$. It makes Pukanszky redundant.) Then $\gamma_{\theta, \sigma}$ is given by holomorphic induction via $\mathfrak{b}$

$$
\gamma_{\theta, \sigma}=\mathfrak{b}-\operatorname{Ind}_{N_{\theta}}^{N} \sigma
$$

(see $[1],[2]$ or $[6]$ ). We shall show that in fact $\mathfrak{b}$ is a polarization for $\varphi$ with satisfactory properties. Then

$$
\pi_{\varphi, \tau}=\mathfrak{b}-\operatorname{Ind}_{G_{\varphi}}^{G} \tau
$$


and by $[2$, p. 116], we conclude

$$
\pi_{\varphi, \tau}=\mathfrak{b}-\operatorname{Ind}_{G \varphi}^{G} \tau=\operatorname{Ind}_{N}^{G}\left[\mathfrak{b}-\operatorname{Ind}_{N_{\theta}}^{N} \sigma\right]=\operatorname{Ind}_{N}^{G} \gamma_{\theta, \sigma}=\pi
$$

To verify the assertion about $\mathfrak{b}$, we start with the equality $\mathfrak{g}_{\varphi}=\mathfrak{n}_{\varphi}$. This subalgebra is co-dimension 1 in $\mathfrak{n}_{\theta}$ (by (2.2)). Thus in the sequence

$$
\left(\mathfrak{g}_{\varphi}\right)_{c} \subset\left(\mathfrak{n}_{\theta}\right)_{c} \subset \mathfrak{b} \subset \mathfrak{n}_{c} \subset \mathfrak{g}_{c}
$$

we have $\operatorname{dim} \mathfrak{b} /\left(\mathfrak{g}_{\varphi}\right)_{c}=\frac{1}{2} \operatorname{dim} \mathfrak{g}_{c} /\left(\mathfrak{g}_{\varphi}\right)_{c}$. Also $\varphi[\mathfrak{b}, \mathfrak{b}]=\theta[\mathfrak{b}, \mathfrak{b}]=0$, so $\mathfrak{b}$ is a polarization for $\varphi$. The positivity of $\mathfrak{b}$ as a polarization for $\varphi$ is automatic from that of $\theta$. Invariance is also clear since $G_{\varphi} \subset G_{\theta}=N_{\theta}$. What about admissibility? If $\mathfrak{u}_{\mathfrak{g}}=\mathfrak{u}_{\mathfrak{n}}$, it is automatic. But the latter may be false (see Example 5 at the end of the section). In fact we have $\mathfrak{u}_{\mathfrak{n}}=\mathfrak{u}_{\mathfrak{g}} \cap \mathfrak{n}$. This is because $\mathfrak{u}_{\mathfrak{n}}$ is a characteristic ideal in $\mathfrak{n} \Rightarrow$ it's an ideal in $\mathfrak{g} \Rightarrow \mathfrak{U}_{\mathfrak{n}} \subset \mathfrak{u}_{\mathfrak{g}}$. But conversely $\mathfrak{n} \cap \mathfrak{u}_{\mathfrak{g}}$ is a nilpotent ideal in $\mathfrak{n} \Rightarrow \mathfrak{n} \cap \mathfrak{u}_{\mathfrak{g}} \subset \mathfrak{u}_{\mathfrak{n}}$. In fact, as Example 5 reveals, $\mathfrak{b}$ may not be admissible with respect to $\mathfrak{u}_{\mathfrak{g}}$. But, by choice, $\mathfrak{b}$ is admissible with respect to $\mathfrak{u}_{\mathfrak{n}}$. The latter is a nilpotent ideal in $\mathfrak{g}$ which satisfies $\mathfrak{g} / \mathfrak{u}_{\mathfrak{n}}$ is abelian. Indeed

$$
[\mathfrak{g}, \mathfrak{g}] \subset \mathfrak{u}_{\mathfrak{g}} \cap \mathfrak{n}=\mathfrak{u}_{\mathfrak{n}}
$$

That is sufficient (by [1]) to conclude

$$
\pi_{\varphi, \tau}=b-\operatorname{Ind}_{G_{\varphi}}^{G} \tau .
$$

We check, finally, that $G \cdot \varphi \cap p^{-1}(N \cdot \theta)=N \cdot \varphi$. This is clear, because if $\left.g \cdot \varphi\right|_{\mathfrak{n}}=n \cdot \theta$, then $n^{-1} g \in G_{\theta}=N_{\theta} \Rightarrow g \in N$. This concludes the proof in case (i). But before proceeding to the next case, we insert some extra structural conclusions not needed until Section 3. Analogs of these conclusions will be needed in each of the remaining three cases. From $N_{\theta}^{0} \cdot \varphi=\varphi+\mathfrak{n}^{\perp} \supset G_{\theta} \cdot \varphi$, it is clear that $G_{\theta}=G_{\varphi} N_{\theta}^{0}$. Also

$$
\begin{gathered}
\mathbf{R} \approx N_{\theta} / N_{\varphi} \approx N_{\theta}^{0} N_{\varphi} / N_{\varphi}=N_{\theta}^{0} / N_{\varphi} \cap N_{\theta}^{0} \Rightarrow N_{\varphi} \cap N_{\theta}^{0} \\
\text { is connected, so } N_{\varphi} \cap N_{\theta}^{0}=N_{\varphi}^{0} .
\end{gathered}
$$

Thus we have the following diagram

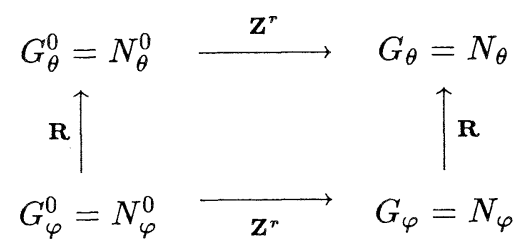

FIgURe 1 
Actually all quotients in the diagram are commutative groups - in fact $\left[G_{\theta}, G_{\theta}\right] \subset$ $G_{\varphi}^{0}$. We leave the verification of that inclusion to the reader - the details being almost identical to the corresponding forthcoming arguments in cases (ii) and (iii).

(ii) Now we suppose $G_{\theta} / N_{\theta} \cong \mathbf{Z}$. Then $G_{\theta} N / N \cong G_{\theta} / N_{\theta} \cong \mathbf{Z}$. In fact $G_{\gamma}=G_{\theta} N$ because - as we shall demonstrate below - $G_{\theta}=\left(G_{\theta}\right)_{\sigma}$. It is a priori possible that $\left(G_{\theta}\right)_{\sigma} \neq G_{\theta}$, but the reasoning below will show this cannot happen. Now

$$
N=\left(G_{\theta} N\right)^{0}=G_{\theta}^{0} N \Rightarrow G_{\theta}^{0} \subset N_{\theta} \Rightarrow G_{\theta}^{0}=N_{\theta}^{0} .
$$

In particular $\mathfrak{g}_{\theta}=\mathfrak{n}_{\theta}$ and $\mathfrak{g}_{\gamma}=\mathfrak{n}$. We choose $\varphi \in p^{-1}(\theta)$ as in (i). Then

$$
N_{\theta}^{0} \cdot \varphi=\varphi+\mathfrak{g}_{\gamma}^{\perp}=\varphi+\mathfrak{n}^{\perp} .
$$

This implies $\operatorname{dim} \mathfrak{n}_{\theta} / \mathfrak{n}_{\varphi}=1$ and there is only one $G$-orbit, $G \cdot \varphi$, lying over $N \cdot \theta$. Next

$$
\mathfrak{n}_{\varphi} \subset \mathfrak{g}_{\varphi} \subset \mathfrak{g}_{\theta}=\mathfrak{n}_{\theta} \Rightarrow \mathfrak{g}_{\varphi}=\mathfrak{n}_{\varphi} \quad \text { or } \quad \mathfrak{g}_{\varphi}=\mathfrak{g}_{\theta} .
$$

But $G_{\theta} \cdot \varphi \supset N_{\theta}^{0} \cdot \varphi=\varphi+\mathfrak{n}^{\perp} \Rightarrow \operatorname{dim} \mathfrak{g}_{\theta} / \mathfrak{g}_{\varphi}=1$. Therefore $\mathfrak{g}_{\varphi}=\mathfrak{n}_{\varphi}$ and $G_{\varphi}^{0}=N_{\varphi}^{0}$. Also $\left.G_{\theta} \cdot \varphi\right|_{\mathfrak{n}}=\theta \Rightarrow G_{\theta} \cdot \varphi \subset \varphi+\mathfrak{n}^{\perp}$. Combining with the above, we have

$$
G_{\theta} \cdot \varphi=\varphi+\mathfrak{n}^{\perp}=N_{\theta}^{0} \cdot \varphi \Rightarrow G_{\theta}=G_{\varphi} N_{\theta}^{0} .
$$

An immediate consequence is $N_{\theta}=N_{\varphi} N_{\theta}^{0}$. So we have a diagram

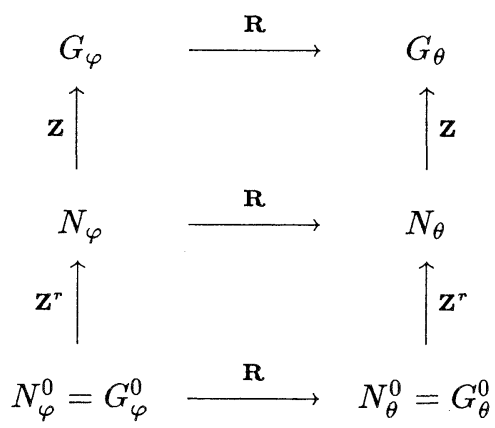

Figure 2

Thus far what is justified in Figure 2 are the two equalities and the isomorphisms: $G_{\theta} / N_{\theta} \cong \mathbf{Z} ; N_{\theta} / N_{\theta}^{0} \cong \mathbf{Z}^{r}$ (we know it's free abelian, let $r$ be the rank); $G_{\theta} / G_{\varphi} \approx \mathbf{R}$ (a priori $\mathfrak{g}_{\varphi}$ might not be an ideal in $\mathfrak{g}_{\theta}$, so the isomorphism $\approx$ means $\mathbf{R}$ is a principal homogeneous space for $G_{\theta}$ with stability group $G_{\varphi}$ ); and similarly $N_{\theta}^{0} / N_{\varphi}^{0} \approx \mathbf{R}$. We justify the remaining assertions:

$$
\begin{aligned}
G_{\theta}=G_{\varphi} N_{\theta}^{0} & \Rightarrow G_{\theta}=G_{\varphi} N_{\theta} \Rightarrow G_{\theta} / N_{\theta} \cong G_{\varphi} / N_{\varphi} ; \\
\mathbf{R} & \approx N_{\theta}^{0} / N_{\varphi}^{0} \approx G_{\theta} / G_{\varphi}=G_{\varphi} N_{\theta}^{0} / G_{\varphi} \cong N_{\theta}^{0} / G_{\varphi} \cap N_{\theta}^{0} \\
& \cong\left(N_{\theta}^{0} / N_{\varphi}^{0}\right) /\left(G_{\varphi} \cap N_{\theta}^{0}\right) / N_{\varphi}^{0} \Rightarrow G_{\varphi} \cap N_{\theta}^{0}=N_{\varphi}^{0} .
\end{aligned}
$$


Therefore

$$
N_{\theta} / N_{\theta}^{0}=N_{\varphi} N_{\theta}^{0} / N_{\theta}^{0} \cong N_{\varphi} / N_{\varphi} \cap N_{\theta}^{0}=N_{\varphi} / N_{\varphi}^{0}
$$

Finally

$$
N_{\theta} / N_{\varphi}=N_{\varphi} N_{\theta}^{0} / N_{\varphi} \approx N_{\theta}^{0} / N_{\varphi} \cap N_{\theta}^{0}=N_{\theta}^{0} / N_{\varphi}^{0} \approx \mathbf{R} .
$$

At last we show all the tildes are actually group isomorphisms. This follows from $\left[G_{\theta}, G_{\theta}\right] \subset G_{\varphi}^{0}$, which we now demonstrate. First we observe that $\left[\mathfrak{n}_{\theta}, \mathfrak{n}_{\theta}\right] \subset \mathfrak{n}_{\varphi}$. In fact

$$
\varphi\left[\left[\mathfrak{n}_{\theta}, \mathfrak{n}_{\theta}\right], \mathfrak{g}\right] \subset \varphi\left[\left[\mathfrak{n}_{\theta}, \mathfrak{g}\right], \mathfrak{n}_{\theta}\right] \subset \theta\left[\mathfrak{n}, \mathfrak{n}_{\theta}\right]=0 .
$$

Thus $\left[N_{\theta}^{0}, N_{\theta}^{0}\right] \subset N_{\varphi}^{0}$. Next I claim $\left[G_{\varphi}, N_{\theta}\right] \subset N_{\varphi}$. Indeed if $n_{\theta} \cdot \varphi=\varphi+s_{0} \alpha$, then $g_{\varphi} n_{\theta} g_{\varphi}^{-1} \cdot \varphi=g_{\varphi} \cdot\left(\varphi+s_{0} \alpha\right)=\varphi+s_{0} \alpha=n_{\theta} \cdot \varphi$ (since $\left.g \cdot \alpha=\alpha, \forall g \in G\right)$. Therefore $\left[G_{\varphi}, N_{\theta}^{0}\right] \subset N_{\theta}^{0} \cap N_{\varphi}=N_{\varphi}^{0}$. We already know $\left[G_{\varphi}, G_{\varphi}\right] \subset G_{\varphi}^{0}$. Combining all these with the equality $G_{\theta}=G_{\varphi} N_{\theta}^{0}$, we get the desired result.

Now let $\tau \in \mathcal{X}(\varphi)$. Since $G_{\varphi} / G_{\varphi}^{0}$ is abelian and $G_{\varphi}^{0} \subset N_{\varphi} \subset G_{\varphi}$, we can, by hitting $\tau$ with a properly chosen character which is trivial on $G_{\varphi}^{0}$, make $\left.\tau\right|_{N_{\varphi}}=\left.\sigma\right|_{N_{\varphi}}$. That is $\mathcal{X}_{\sigma}(\varphi) \neq \emptyset$. In fact it is obvious from Figure 2 that

$$
\mathcal{X}_{\sigma}(\varphi) \approx\left(G_{\varphi} / N_{\varphi}\right)^{\wedge}, \text { a } 1 \text {-torus. }
$$

Next define a character $\bar{\sigma}$ of $G_{\theta}$ by the formula $\bar{\sigma}\left(g_{\varphi} n_{\theta}^{0}\right)=\tau\left(g_{\varphi}\right) \chi_{\theta}\left(n_{\theta}^{0}\right) . \bar{\sigma}$ is welldefined since $\left.\tau\right|_{G_{\varphi} \cap N_{\theta}^{0}}=\left.\sigma\right|_{G_{\varphi} \cap N_{\theta}^{0}}=\left.\chi_{\varphi}\right|_{N_{\varphi}^{0}}$. Moreover $\bar{\sigma}$ is a character since $G_{\varphi}$ fixes $\chi_{\theta}$. But since $G_{\theta} / G_{\varphi}^{0}$ is abelian, $\bar{\sigma}$ can be adjusted - without changing its value on $N_{\theta}^{0}$ - so that $\left.\bar{\sigma}\right|_{N_{\theta}}=\sigma$. This implies, since $\sigma$ extends as a character to $G_{\theta}$, that $\left(G_{\theta}\right)_{\sigma}=G_{\theta}$. Hence $G_{\gamma}=\left(G_{\theta}\right)_{\sigma} N$ and $G_{\gamma} / N \cong G_{\theta} / N_{\theta} \cong \mathbf{Z}$ as asserted earlier. Formula (2.1) becomes

$$
\pi=\operatorname{Ind}_{N}^{G} \gamma_{\theta, \sigma}=\int_{\left(G_{\theta} / N_{\theta}\right)^{\wedge}}^{\oplus} \operatorname{Ind}_{G_{0} N}^{G} \rho \tilde{\gamma} d \rho .
$$

We shall show that the representations $\pi_{\varphi, \tau}, \varphi$ as above, $\tau \in \mathcal{X}_{\sigma}(\varphi)$, constitute precisely the constituents of the direct integral (2.3). Choose a polarization $\mathfrak{b}$ for $\theta$ with all the requisite properties to construct $\gamma_{\theta, \sigma}=\mathfrak{b}-\operatorname{Ind}_{N_{\theta}}^{N} \sigma$. Then exactly as in case (i), it may be shown that $\mathfrak{b}$ is a positive polarization for $\varphi$, admissible with respect to $\mathfrak{U}_{\mathfrak{n}}$. What is not immediately clear is that $\mathfrak{b}$ is $G_{\varphi}$-invariant. In fact an adaptation of $[1, \S I I .3]$ shows that this can be assumed. (More precisely, we have to repeat the proof of $\left[1\right.$, Theorem 1.3 .2$, p. 304] to construct $\mathfrak{b}=\mathfrak{b}_{1}+\mathfrak{b}_{2}$ using: $F=\operatorname{Ad}_{\mathfrak{u}_{\mathfrak{n}}} G_{\theta}$ and $[\mathfrak{g}, \mathfrak{g}] \subset \mathfrak{U}_{\mathfrak{n}}$ to get $G_{\varphi}$-invariance of $\mathfrak{b}_{1}=\mathfrak{b} \cap\left(\mathfrak{u}_{\mathfrak{n}}\right)_{c}$; and then use $[\mathfrak{g}, \mathfrak{g}] \subset \mathfrak{n}$ to get invariance of $\mathfrak{b}_{2}$ (in the nilpotent $\mathfrak{m} / \mathfrak{p}$ in the notation of $[1]$, $\left.\mathfrak{m}=\mathfrak{n}_{\theta}\right)$.) But the following sequence of equivalences is evident

$$
\begin{gathered}
\pi=\operatorname{Ind}_{N}^{G} \gamma_{\theta, \sigma}=\operatorname{Ind}_{N}^{G} \mathfrak{b}-\operatorname{Ind}_{N_{\theta}}^{N} \sigma=\mathfrak{b}-\left.\operatorname{Ind}_{N_{\varphi}}^{G} \sigma\right|_{N_{\varphi}}=\mathfrak{b}-\operatorname{Ind}_{G_{\varphi}}^{G} \int_{\mathcal{X}_{\sigma}(\varphi)}^{\oplus} \tau d \tau \\
=\int_{\mathcal{X}_{\sigma}(\varphi)}^{\oplus} \mathfrak{b}-\operatorname{Ind}_{G_{\varphi}}^{G} \tau d \tau=\int_{\mathcal{X}_{\sigma}(\varphi)}^{\oplus} \pi_{\varphi, r} d \tau .
\end{gathered}
$$


As in (i) we finish with the demonstration that $G \cdot \varphi \cap p^{-1}(N \cdot \theta)=N \cdot \varphi$. In fact $\left.g \cdot \varphi\right|_{\mathfrak{n}}=n \cdot \theta \Rightarrow n^{-1} g \in G_{\theta}=G_{\varphi} N_{\theta}^{0}=N_{\theta}^{0} G_{\varphi} \Rightarrow g \cdot \varphi \in N \cdot \varphi$. This concludes the proof of case (ii) in Theorem 1 .

(iii) $G_{\theta} / N_{\theta} \cong \mathbf{R}$ and $G_{\theta}=\left(G_{\theta}\right)_{\sigma}$. Now $G=G_{\theta} N_{\theta} \Rightarrow G=G_{\theta}^{0} N \Rightarrow G_{\theta}=$ $G_{\theta}^{0} N_{\theta}$. Also $\mathfrak{g}=\mathfrak{g}_{\theta}+\mathfrak{n}=\mathfrak{g}_{\gamma}$. Choose $\varphi \in p^{-1}(\theta), \varphi(X)=0$ as usual. Then

$$
N_{\theta}^{0} \cdot \varphi=\varphi+\mathfrak{g}_{\gamma}^{\perp}=\varphi \Rightarrow N_{\theta}^{0}=N_{\varphi}^{0} .
$$

Continuing, $\operatorname{dim} \mathfrak{g}_{\theta} / \mathfrak{n}_{\theta}=1$ and $\mathfrak{n}_{\theta}=\mathfrak{n}_{\varphi} \subset \mathfrak{g}_{\varphi} \subset \mathfrak{g}_{\theta} \Rightarrow \mathfrak{g}_{\varphi}=\mathfrak{n}_{\varphi}$ or $\mathfrak{g}_{\varphi}=\mathfrak{g}_{\theta}$. But

$$
\operatorname{dim} \mathfrak{g} / \mathfrak{g}_{\varphi} \leq \operatorname{dim} \mathfrak{g} / \mathfrak{g}_{\theta}+1=\operatorname{dim} \mathfrak{g} / \mathfrak{n}_{\theta}=\operatorname{dim} \mathfrak{n} / \mathfrak{n}_{\theta}+1
$$

Since co-adjoint orbits have even dimension, we conclude

$$
\operatorname{dim} \mathfrak{g} / \mathfrak{g}_{\varphi}=\operatorname{dim} \mathfrak{n} / \mathfrak{n}_{\theta} \Rightarrow \operatorname{dim} \mathfrak{g}_{\varphi}=\operatorname{dim} \mathfrak{n}_{\theta}+1=\operatorname{dim} \mathfrak{g}_{\theta}
$$

Hence

$$
\mathfrak{g}_{\varphi}=\mathfrak{g}_{\theta} \quad G_{\varphi}^{0}=G_{\theta}^{0} \quad G_{\theta}=G_{\varphi}^{0} N_{\theta} .
$$

Now we have the following diagram

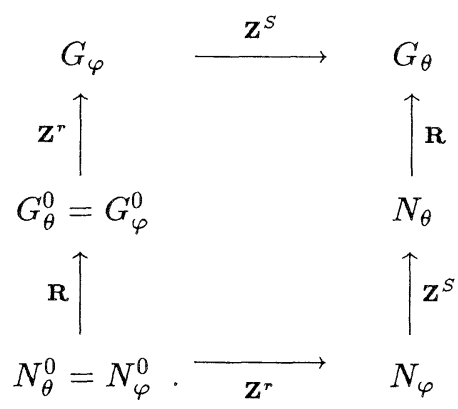

FIGURE i

What is justified thus far are the two equalities and the isomorphisms: $G_{\theta} / N_{\theta} \cong \mathbf{R}$ and $G_{\theta}^{0} / N_{\theta}^{0} \cong \mathbf{R}$. Actually the hypothesis $G_{\theta}=\left(G_{\theta}\right)_{\sigma}$ will force some drastic simplifications in Figure i, namely $s=0$. But before seeing that, we justify the three unverified assertions. In fact

$$
G_{\theta}=G_{\varphi}^{0} N_{\theta} \Rightarrow G_{\theta}=G_{\varphi} N_{\theta} \Rightarrow \mathbf{R} \approx G_{\theta} / N_{\theta} \cong G_{\varphi} N_{\theta} / N_{\theta} \cong G_{\varphi} / N_{\varphi} .
$$

Moreover

$$
\begin{aligned}
\mathbf{R} & \approx G_{\varphi} / N_{\varphi} \cong G_{\varphi}^{0} / N_{\varphi}^{0} \cong G_{\theta}^{0} / N_{\theta}^{0} \cong G / N \\
& \cong G_{\theta} N / N \cong G_{\theta} / N_{\theta} \cong G_{\varphi}^{0} N_{\theta} / N_{\theta} \cong G_{\varphi}^{0} /\left(N_{\theta} \cap G_{\varphi}^{0}\right) \\
& \cong\left(G_{\varphi}^{0} / N_{\varphi}^{0}\right) /\left(N_{\theta} \cap G_{\varphi}^{0}\right) / N_{\varphi}^{0} \Rightarrow G_{\varphi}^{0} \cap N_{\varphi}=N_{\varphi}^{0}
\end{aligned}
$$


and therefore

$$
G_{\varphi} / G_{\varphi}^{0} \cong G_{\varphi}^{0} N_{\varphi} / G_{\varphi}^{0} \cong N_{\varphi} / N_{\varphi}^{0} .
$$

A completely analogous set of equations shows

$$
G_{\theta} / G_{\theta}^{0} \cong N_{\theta} / N_{\theta}^{0}
$$

Finally we show that - as in case (ii) - all quotients in Figure i are abelian groups, that is $\left[G_{\theta}, G_{\theta}\right] \subset N_{\theta}^{0}$. This follows from $G_{\theta}=G_{\theta}^{0} N_{\theta}$ and the three assertions:

$$
[G, G] \subset N \Rightarrow\left[G_{\theta}^{0}, G_{\theta}^{0}\right] \subset N_{\theta}^{0}
$$

$\left[N_{\theta}, N_{\theta}\right] \subset N_{\theta}^{0}$, since $N$ is type $I \Rightarrow N_{\theta} / N_{\theta}^{0}$ is abelian;

$\left[G_{\theta}^{0}, N_{\theta}\right] \subset N_{\theta} \cap G_{\theta}^{0}=N_{\theta}^{0}$, since both $N_{\theta}$ and $G_{\theta}^{0}$ are normal in $G_{\theta}$.

Now we bring in the hypothesis $G_{\theta}=\left(G_{\theta}\right)_{\sigma}$. We begin by observing that, since $G_{\theta} / N_{\theta} \cong \mathbf{R}$ supports no Mackey obstructions, the character $\sigma$ can be extended to a character $\bar{\sigma}$ of $G_{\theta}$. Since $G_{\theta} / N_{\theta}^{0}$ is abelian, we can hit $\bar{\sigma}$ with a character, trivial on $N_{\theta}^{0}$, so that the result, say $\overline{\bar{\sigma}}$, satisfies

$$
\left.\overline{\bar{\sigma}}\right|_{G_{\varphi}^{0}}=\chi_{\varphi} .
$$

Let $n_{\theta} \in N_{\theta}, g_{\varphi}^{0} \in G_{\varphi}^{0}$. Then

$$
\begin{aligned}
& \overline{\bar{\sigma}}\left(n_{\theta} g_{\varphi}^{0} n_{\theta}^{-1}\right)=\bar{\sigma}\left(g_{\varphi}^{0}\right)=\chi_{\varphi}\left(g_{\varphi}^{0}\right)=\chi_{\varphi}\left(n_{\theta} g_{\varphi}^{0} n_{\theta}^{-1}\right) \\
& \Rightarrow n_{\theta} \cdot \varphi=\varphi \quad \text { on } \quad \mathfrak{g}_{\varphi}=\mathfrak{g}_{\theta} .
\end{aligned}
$$

But since $\mathfrak{g}=\mathfrak{g}_{\theta}+\mathfrak{n}$, we have $n_{\theta} \cdot \varphi=\varphi$, i.e. $N_{\theta}=N_{\varphi}$. Then $G_{\theta}=G_{\theta}^{0} N_{\theta}=$ $G_{\varphi}^{0} N_{\varphi}=G_{\varphi}$. This forces $s=0$ in Figure i, and the diagram simplifies to

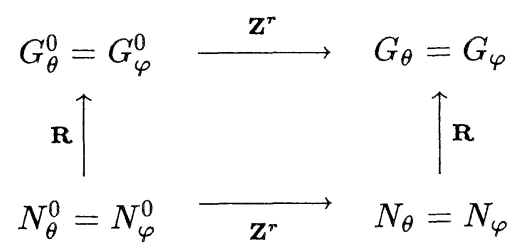

FIGURE 3

Now choose $\varphi \in p^{-1}(\theta)$ as usual, i.e. $\varphi(X)=0,\left.\varphi\right|_{\mathfrak{n}}=\theta$. Suppose that for some $g \in G$, we have $g \cdot \varphi=\varphi+s \alpha, s \in \mathbf{R}$. Restricting both sides to $\mathfrak{n}$ we obtain $g \in G_{\theta}=G_{\varphi} \Rightarrow s=0$. That is the points $\{\varphi+s \alpha: s \in \mathbf{R}\}$ all lie in distinct $G$ orbits. Now change notation momentarily and let $\varphi$ denote any element in $p^{-1}(\theta)$. It is clear that everything done so far in case (iii) applies to any such $\varphi$. In fact, 
since $G_{\varphi}=G_{\varphi}^{0} N_{\varphi}=G_{\varphi}^{0} N_{\theta}$, the set $\mathcal{X}_{\sigma}(\varphi)$ contains at most one element. It is actually non-empty since the prescription

$$
\tau\left(g_{\varphi}^{0} n_{\theta}\right)=\chi_{\varphi}\left(g_{\varphi}^{0}\right) \sigma\left(n_{\theta}\right)
$$

is well-defined and yields a member of $\mathcal{X}_{\sigma}(\varphi)$.

Now we know $G_{\gamma}=G$ and

$$
\pi=\operatorname{Ind}_{N}^{G} \gamma_{\theta, \sigma}=\int_{(G / N)^{\wedge}}^{\oplus} \operatorname{Ind}_{G_{\theta} N}^{G} \rho \tilde{\gamma} d \rho
$$

Reverting to the choice $\varphi \in p^{-1}(\theta), \varphi(X)=0$, we shall show that the representations $\pi_{\varphi+s \alpha, r_{s}}$ are the constituents of the direct integral (2.4). As usual a careful choice of polarization is required. We start with an admissible polarization $\mathfrak{b}_{1}$ for $\theta$. Exactly as in part (ii) we can choose it so that it is $G_{\varphi}$-invariant. Then we set

$$
\mathfrak{b}=\mathfrak{b}_{1}+\left(\mathfrak{g}_{\varphi}\right)_{c}
$$

It is routine to check that $\mathfrak{b}$ is an invariant positive polarization for $\varphi$ which is admissible with respect to $\mathfrak{U}_{\mathfrak{n}}$. Therefore

$$
\pi_{\varphi+s \alpha, \tau_{S}}=\mathfrak{b}-\operatorname{Ind}_{G_{\varphi}}^{G} \tau_{s} .
$$

Now the character $\chi_{\varphi+s \alpha}$ splits into $\chi_{\varphi+s \alpha}=\chi_{\varphi} \chi_{s \alpha}$. Here $\chi_{s \alpha}$ is the unique unitary character of $G$, trivial on $N$, satisfying $\chi_{s \alpha}(\exp t X)=e^{i s t}$. It is therefore also clear that

$$
\pi_{\varphi+s \alpha, \tau_{S}}=\chi_{s \alpha} \pi_{\varphi, \tau} .
$$

Thus to prove these representations agree with the representations in the direct integral (2.4), it is enough to verify that $\pi_{\varphi,\left.\tau\right|_{N}} \cong \gamma_{\theta, \sigma}$. In fact it is obvious that

$$
\pi_{\varphi,\left.\tau\right|_{N}}=\left.\left(\mathfrak{b}-\operatorname{Ind}_{G_{\varphi}}^{G} \tau_{s}\right)\right|_{N}=\mathfrak{b}_{1}-\operatorname{Ind}_{N_{\theta}}^{N} \sigma=\gamma_{\theta, \sigma}
$$

Finally we have $G \cdot(\varphi+s \alpha) \cap p^{-1}(N \cdot \theta)=N \cdot(\varphi+s \alpha)$ because $\left.g \cdot(\varphi+s \alpha)\right|_{\mathfrak{n}}=$ $n \cdot \theta \Rightarrow n^{-1} g \in G_{\theta}=G_{\varphi}$. Hence, as in (i) and (ii), this proves the formula

$$
\operatorname{Ind}_{N}^{G} \gamma_{\theta, \sigma}=\int_{p^{-1}(N \cdot \theta) / N}^{\oplus} \int_{\mathcal{X}_{\sigma}(\varphi)}^{\oplus} \pi_{\varphi, \tau} d \tau d \dot{\varphi} .
$$

(iv) $G_{\theta} / N_{\theta} \cong \mathbf{R}$ and $G_{\theta} \neq\left(G_{\theta}\right)_{\sigma}$. There are two possibilities: either $\left(G_{\theta}\right)_{\sigma} /$ $N_{\theta} \cong \mathbf{Z}$ or $\left(G_{\theta}\right)_{\sigma}=N_{\theta}$. We shall prove momentarily that it must be the former. Now everything we did in case (iii) remains valid until the moment we looked at $\left(G_{\theta}\right)_{\sigma}$. In particular Figure $\mathrm{i}$ is valid - but with $s=1$, as we shall demonstrate momentarily. 


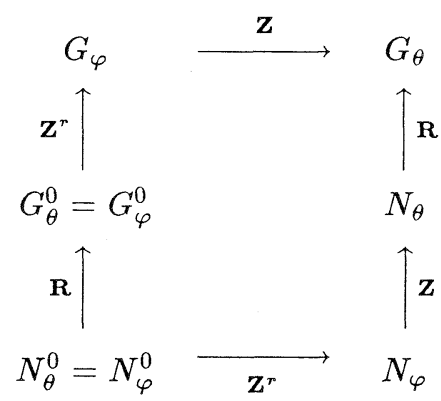

FIGURE 4

It is clear (from $G_{\theta}^{0}=G_{\varphi}^{0}$ ) that $G_{\theta} / G_{\varphi}$ is discrete - it is an abelian group since we know $\left[G_{\theta}, G_{\theta}\right] \subset N_{\varphi}^{0}$. Similarly for $N_{\theta} / N_{\varphi}$. Now if $N_{\theta}=N_{\varphi}$, then the equality $G_{\theta}=G_{\theta}^{0} N_{\theta}=G_{\varphi}^{0} N_{\varphi}$ insures that the map

$$
g_{\varphi}^{0} n_{\varphi} \longrightarrow \chi_{\varphi}\left(g_{\varphi}^{0}\right) \sigma\left(n_{\varphi}\right)
$$

is a character of $G_{\theta}$ that extends $\sigma$. This contradicts the assumption $G_{\theta} \neq\left(G_{\theta}\right)_{\sigma}$. Therefore $N_{\theta} \neq N_{\varphi}$. Now we use the fact that $G$ type I implies its coadjoint orbits are locally closed [1]. It follows that the orbits $N_{\theta} \cdot \varphi=\left(N_{\theta} / N_{\theta}^{0}\right) \cdot \varphi$ must be discrete on the line $p^{-1}(\theta)$. Choose $s_{0}$ the smallest positive real such that $\varphi+s_{0} \alpha \in N_{\theta} \cdot \varphi$. Then

$$
N_{\theta} \cdot \varphi=\varphi+s_{0} \mathbf{Z} \alpha .
$$

That is $N_{\theta} / N_{\varphi} \cong \mathbf{Z}$. Also $G_{\theta} / G_{\varphi}=G_{\varphi} N_{\theta} / G_{\varphi}=N_{\theta} / N_{\varphi} \cong \mathbf{Z}$. So all the details of Figure 4 have been corroborated. We also have that the $G$-orbits $G \cdot(\varphi+s \alpha)$, $0 \leq s<s_{0}$ constitute the distinct $G$-orbits lying over $N \cdot \theta$.

Now we continue as in (iii). We change notation temporarily so that $\varphi$ is any element of $p^{-1}(\theta)$. The equality $G_{\varphi}=G_{\varphi}^{0} N_{\varphi}$ says that the set $\mathcal{X}_{\sigma}(\varphi)$ contains at most one element. In fact there is exactly one, namely $\tau\left(g_{\varphi}^{0} n_{\varphi}\right)=\chi_{\varphi}\left(g_{\varphi}^{0}\right) \sigma\left(n_{\varphi}\right)$. (Note in this case $\tau$ does not extend to $G_{\theta}$.) Now we have $G_{\gamma} / N \cong \mathbf{Z}$ and

$$
\pi=\operatorname{Ind}_{N}^{G} \gamma_{\theta, \sigma}=\int_{\left(G_{\gamma} / N\right)^{\wedge}}^{\oplus} \operatorname{ind}_{G_{\theta} N}^{G} \rho \tilde{\gamma} d \rho .
$$

As usual we have to recognize the representations in (2.5) orbitally. We proceed as in (iii): take $\mathfrak{b}$ a positive admissible $G_{\varphi}$-invariant polarization for $\theta$ and form $\mathfrak{b}=\mathfrak{b}_{1}+\left(\mathfrak{g}_{\varphi}\right)_{c}$. This will be an invariant positive admissible polarization for any $\varphi+s \alpha, 0 \leq s<s_{0}, \varphi \in p^{-1}(\theta), \varphi(X)=0$. As in (iii),

$$
\pi_{\varphi+s \alpha, \tau_{S}}=\chi_{s \alpha} \pi_{\varphi, \tau}
$$


This time therefore it is enough to show

$$
\pi_{\varphi,\left.\tau\right|_{N}}=\int_{G / G_{\gamma}}^{\oplus} g \cdot \gamma_{\theta, \sigma} d \dot{g}
$$

(see [5]). We have $G=G_{\theta} N \Rightarrow G=G_{\theta}^{0} N=G_{\varphi}^{0} N$. Therefore

$$
G_{\gamma}=\left(G_{\varphi}^{0}\right)_{\gamma} N=\left(G_{\varphi}^{0}\right)_{\sigma} N
$$

Thus to prove (2.6), it suffices to show

$$
\pi_{\varphi, \tau \mid N}=\int_{G_{\varphi}^{0} /\left(G_{\varphi}^{0}\right)_{\sigma}}^{\oplus} g \cdot \gamma_{\theta, \sigma} d \dot{g} .
$$

Clearly, for $g \in G_{\varphi}$, we have $g \cdot \gamma_{\theta, \sigma}=\gamma_{\theta, g \cdot \sigma}$. Moreover we have

Lemma. Let $\rho=\left.\sigma\right|_{N_{\varphi}}$. Then $\operatorname{Ind}_{N_{\varphi}}^{N_{\theta}} \rho=\int_{G_{\varphi}^{0} /\left(G_{\varphi}^{0}\right)_{\sigma}}^{\oplus} g \cdot \sigma d \dot{g}$.

Proof. Since $\rho$ extends to a character of $N_{\theta}$, namely $\sigma$, it is obvious that

$$
\operatorname{Ind}_{N_{\varphi}}^{N_{\theta}} \rho=\int_{\mathcal{X}_{\rho}(\theta)}^{\oplus} \sigma_{1} d \sigma_{1}
$$

where $\mathcal{X}_{\rho}(\theta)=\left\{\sigma_{1} \in \widehat{N}_{\theta}:\left.\sigma_{1}\right|_{N_{\varphi}}=\rho=\left.\tau\right|_{N_{\varphi}}\right\}$. Now obviously $G_{\varphi}^{0}$ acts as a group of transformations on $\mathcal{X}_{\rho}(\theta)$. Consider the orbit $\mathcal{O}_{\sigma}$ of $\sigma$. It will be connected. But

$$
\mathcal{X}_{\rho}(\theta) \approx\left(N_{\theta} / N_{\varphi}\right)^{\wedge}, \text { a 1-torus. }
$$

Thus either the orbit $\mathcal{O}_{\sigma}$ is everything or only a point. It cannot be a point since $G_{\varphi}^{0} \neq\left(G_{\varphi}^{0}\right)_{\sigma}$ (a consequence of $\left.G_{\theta} \neq\left(G_{\theta}\right)_{\sigma}\right)$. Hence $G_{\varphi}^{0} /\left(G_{\varphi}^{0}\right)_{\sigma} \approx \mathcal{X}_{\rho}(\theta)$ and the lemma is proven.

The following result - which I do not need in this paper - is an interesting consequence of the Lemma.

Corollary. $\operatorname{Ind}_{G_{\varphi}}^{G_{\theta}} \tau \cong \operatorname{Ind}_{N_{\theta}}^{G_{\theta}} \sigma$.

I leave the actual proof to the reader - remarking only that the right side is determined by [5], and that the resulting direct integral agrees with the left follows from: $G_{\theta}=G_{\varphi} N_{\theta}$ and applications of the Subgroup Theorem and the Lemma to the left side. 
Continuing with the argument in case (iv) we have

$$
\begin{aligned}
\left.\pi_{\varphi, \tau}\right|_{N} & =\left.\left[\mathfrak{b}-\operatorname{Ind}_{G_{\varphi}}^{G} \tau\right]\right|_{N}=\mathfrak{b}-\left.\operatorname{Ind}_{N_{\varphi}}^{N} \tau\right|_{N_{\varphi}} \\
& \cong \mathfrak{b}-\operatorname{Ind}_{N \theta}^{N} \operatorname{Ind}_{N_{\varphi}}^{N_{\theta}} \rho \cong \mathfrak{b}-\operatorname{Ind}_{N_{\theta}}^{N} \int_{G_{\varphi}^{0} /\left(G_{\varphi}^{0}\right)_{\sigma}}^{\oplus} g \cdot \sigma d \dot{g} \\
& \cong \int_{G_{\varphi}^{0} /\left(G_{\varphi}^{0}\right)_{\sigma}}^{\oplus} \mathfrak{b}-\operatorname{Ind}_{N_{\theta}}^{N} g \cdot \sigma d \dot{g}=\int_{G_{\varphi}^{0} /\left(G_{\varphi}^{0}\right)_{\sigma}}^{\oplus} \gamma_{\theta, g \cdot \sigma} d \dot{g} \\
& =\int_{G_{\varphi} /\left(G_{\varphi}^{0}\right)_{\sigma}}^{\oplus} g \cdot \gamma_{\theta, \sigma} d \dot{g} .
\end{aligned}
$$

The argument is concluded by observing as usual that $G \cdot(\varphi+s \alpha) \cap p^{-1}(N \cdot \theta)=$ $N \cdot(\varphi+s \alpha), 0 \leq s<s_{0}$. (This uses $n^{-1} g \in G_{\theta}=G_{\varphi} N_{\theta}$.) The proof of Theorem 1 is therefore complete.

REMARK. We note that the condition which distinguishes between cases (iii) and (iv) really does not depend on $\sigma$. The proof of Theorem 1 shows that (iii) occurs iff $G_{\theta} / N_{\theta} \cong \mathbf{R}$ and $G_{\theta}=G_{\varphi}$, whereas (iv) occurs iff $G_{\theta} / N_{\theta} \cong \mathbf{R}$ and $G_{\theta} \neq G_{\varphi}$, $\varphi \in p^{-1}(\theta)$.

We now illustrate that each of the four cases of Theorem 1 does occur.

ExAmples. In every case $G$ is the simply connected solvable Lie group corresponding to the Lie algebra $\mathfrak{g}$ generated by the basis and bracket relations listed.

(i) $\mathfrak{g}=\operatorname{sp}\{X, Y, Z\} ;[X, Y]=Z$ (the Heisenberg Lie algebra)

$$
\begin{aligned}
& \mathfrak{n}=\operatorname{sp}\{X, Z\}, \theta=Z^{*}, G_{\theta}=N_{\theta} \\
& \operatorname{Ind}_{N}^{G} \gamma_{\theta}=\pi_{\varphi}, \varphi=Z^{*} .
\end{aligned}
$$

(ii) $\mathfrak{g}=\operatorname{sp}\{T, X, Y\} ;[T, X]=Y,[T, Y]=-X$

(the Euclidean motion Lie algebra)

$$
\begin{aligned}
& \mathfrak{n}=\operatorname{sp}\{X, Y\}, \theta=X^{*}, N_{\theta}=N, G_{\theta}=(\exp 2 \pi \mathbf{Z} T) N \\
& \varphi=X^{*}, G_{\varphi}=(\exp 2 \pi \mathbf{Z} T)(\exp \mathbf{R} X) \\
& \mathcal{X}_{\sigma}(\varphi)=\left\{\chi_{\rho} \chi_{\theta}: \rho \in[0,1)\right\}, \chi_{\rho}(\exp 2 \pi n T)=e^{2 \pi \operatorname{in} \rho} \\
& \operatorname{Ind}_{N}^{G} \gamma_{\theta}=\int_{[0,1)}^{\oplus} \operatorname{Ind}_{G_{\theta}}^{G} \chi_{\rho} \chi_{\theta} d \rho=\int_{\mathcal{X}_{\sigma}(\varphi)}^{\oplus} \pi_{\varphi, \tau} d \tau .
\end{aligned}
$$

(iii) $\mathfrak{g}=\operatorname{sp}\{T, X, Y, Z\} ;[T, X]=X,[T, Y]=-Y,[X, Y]=Z$ 


$$
\begin{aligned}
& \mathfrak{n}=\operatorname{sp}\{X, Y, Z\}, \theta=Z^{*}, N_{\theta}=\exp \mathbf{R} Z, G_{\theta}=(\exp \mathbf{R} T) N_{\theta} \\
& \sigma=1, G_{\theta}=\left(G_{\theta}\right)_{\sigma} \\
& \varphi=Z^{*}, \alpha=T^{*}, \varphi+s \alpha \text { lie in distinct } G \text {-orbits for distinct } s \in \mathbf{R} \\
& \operatorname{Ind}_{N}^{G} \gamma_{\theta}=\int_{\mathbf{R}}^{\oplus} \pi_{\varphi+s \alpha} d s .
\end{aligned}
$$

(iv) $\mathfrak{g}=\operatorname{sp}\{T, X, Y, U, V\} ;[T, X]=Y,[T, Y]=-X,[T, U]=V$

$\mathfrak{n}=\operatorname{sp}\{T, X, Y, V\}, \varphi=\theta=X^{*}+V^{*}, \alpha=U^{*}$

$\mathfrak{n}_{\theta}=\mathbf{R} X+\mathbf{R} V, N_{\theta}=C \exp \mathfrak{n}_{\theta}, C=\exp 2 \pi \mathbf{Z} T$

$\mathfrak{g}_{\theta}=\mathfrak{n}_{\theta}+\mathbf{R}(Y+U), G_{\theta}=C \exp \mathfrak{g}_{\theta}, G_{\theta} / N_{\theta} \cong \mathbf{R}, G_{\varphi}=G_{\theta}^{0}$

$\sigma \in \mathcal{X}(\theta), \sigma(\exp 2 \pi n T \exp W)=e^{2 \pi i m n} \chi_{\theta}(W), W \in \mathfrak{n}_{\theta}, m \in \mathbf{Z}$

$\exp \lambda(Y+U) \in G_{\theta} \Rightarrow \lambda \in \mathbf{Z}$

$\operatorname{Ind}_{N}^{G} \gamma_{\theta, \sigma}=\int_{[0,1)}^{\oplus} \pi_{\varphi+\rho \alpha, \tau_{\rho}} d \rho$.

Finally, here is an example to illustrate the point about nilradicals made in case (i).

(v) $\mathfrak{g}=\operatorname{sp}\{T, X, Y, Z, W\} ;[T, X]=Y,[T, Y]=-X,[X, Y]=Z,[T, W]=Z$

$$
\begin{aligned}
& \mathfrak{n}=\operatorname{sp}\{T, X, Y, Z\}, \varphi=\theta=Y^{*}+Z^{*}, \mathfrak{n}_{\theta}=\mathbf{R} Z+\mathbf{R}(T-Y), \mathfrak{g}_{\varphi}=\mathbf{R} Z \\
& \mathfrak{u}_{\mathfrak{n}}=\operatorname{sp}\{X, Y, Z\}, \mathfrak{u}_{\mathfrak{g}}=\operatorname{sp}\{X, Y, Z, W\} .
\end{aligned}
$$

$\mathfrak{b}=\mathbf{C n}_{\theta}+\mathbf{C}(X+i Y)$ is a positive polarization for both $\theta$ and $\varphi$, admissible with respect to $\mathfrak{u}_{\mathfrak{n}}$. But it is not admissible with respect to $\mathfrak{u}_{\mathfrak{g}}$, since $\mathfrak{b} \cap\left(\mathfrak{u}_{\mathfrak{g}}\right)_{c}=\mathfrak{b} \cap\left(\mathfrak{u}_{\mathfrak{n}}\right)_{c}$ is not a polarization for $\left.\varphi\right|_{\mathfrak{u}_{\mathfrak{g}}}$ (it does not include $W \in\left(\mathfrak{u}_{\mathfrak{g}}\right)_{\varphi \mid \mathfrak{u}_{\mathfrak{g}}}$ ).

\section{The orbital decomposition}

We now pass to the main order of business - the proof of Theorem 2 . We have $H \subset G$ where $G$ is a simply connected type I solvable Lie group and $H$ is closed, connected and contained in the nilradical of $G$. We postulate the existence of a sequence of closed connected type I subgroups

$$
H=G_{0} \subset G_{1} \subset \cdots \subset G_{n-1} \subset G_{n}=G
$$

satisfying $G_{i-1} \triangleleft G_{i}$ and $\operatorname{dim} G_{i} / G_{i-1}=1,1 \leq i \leq n$. Let $\psi \in \mathfrak{h}^{*}, \nu=\nu_{\psi} \in \widehat{H}$. We 
must prove

$$
\begin{aligned}
\operatorname{Ind}_{H}^{G} \nu_{\psi} & =\int_{p^{-1}(H \cdot \psi) / H}^{\oplus} \int_{\mathcal{X}(\varphi)}^{\oplus} \pi_{\varphi, \tau} d \tau d \dot{\varphi} \\
& =\int_{G \cdot p^{-1}(H \cdot \psi) / G}^{\oplus} \int_{\mathcal{X}(\varphi)}^{\oplus} \pi_{\varphi, \tau} d \tau d \ddot{\varphi}
\end{aligned}
$$

The second equation follows immediately from the first. The proof of the first that is of $(3.1)$ - is by induction on $\operatorname{dim} G / H$. If that dimension is one, the theorem is proven already as Theorem 1 . We assume therefore higher co-dimension for $G / H$ and suppose by induction that formula (3.1) is proven for all homogeneous spaces of lower co-dimension. We consider $N=G_{n-1}$. By the induction assumption, Theorem 2 is true for $\operatorname{Ind}_{H}^{N} \nu_{\psi}$. If we combine that with the theorem of induction in stages, and with the commutativity of direct integrals and induced representations, we obtain

$$
\operatorname{Ind}_{H}^{G} \nu_{\psi}=\operatorname{Ind}_{N}^{G} \operatorname{Ind}_{H}^{N} \nu_{\psi}=\operatorname{Ind}_{N}^{G} \int_{p_{\mathfrak{n}, \mathfrak{h}}^{-1}(H \cdot \psi) / H}^{\oplus} \int_{\mathcal{X}(\theta)}^{\oplus} \gamma_{\theta, \sigma} d \sigma d \dot{\theta}
$$

$$
=\int_{p_{\mathfrak{n}, \mathfrak{h}}^{-1}(H \cdot \psi) / H}^{\oplus} \int_{\mathcal{X}(\theta)}^{\oplus} \operatorname{Ind}_{N}^{G} \gamma_{\theta, \sigma} d \sigma d \dot{\theta} \stackrel{?}{=} \int_{p_{\mathfrak{g}, \mathfrak{h}}^{-1}(H \cdot \psi) / H}^{\oplus} \int_{\chi(\varphi)}^{\oplus} \pi_{\varphi, \tau} d \tau d \dot{\varphi}
$$

It remains to prove the final equality (3.2). That means (see e.g. the discussion in $[8, \S 2])$ we must prove equality of spectrum, multiplicity and spectral measure. The first is fairly routine - we take case of it momentarily. The second is hard and constitutes the crux of the argument. The third is intermediate in difficulty. The reader can find a model for the structure of the whole argument in [8], although the details here are very different.

Spectrum. In this subsection (only), we alter the terminology for describing the orbital parameters. The following is Kirillov's terminology. $G$ acts on the set of ordered pairs

$$
\mathcal{R}_{\mathfrak{g}}=\left\{(\varphi, \tau): \varphi \in \mathfrak{g}^{*}, \tau \in \mathcal{X}(\varphi)\right\} .
$$

A $G$-orbit in $\mathcal{R}_{\mathfrak{g}}$ is called a rigged orbit. Now for $(\theta, \sigma) \in \mathcal{R}_{\mathfrak{n}}$, write

$$
q^{-1}(\theta, \sigma)=\left\{(\varphi, \tau) \in \mathcal{R}_{\mathfrak{g}}:\left.\varphi\right|_{\mathfrak{n}}=\theta,\left.\tau\right|_{N_{\varphi}}=\left.\sigma\right|_{N_{\varphi}}\right\}
$$

Then Theorem 1 asserts that, regardless of the structure of $\operatorname{Ind}_{N}^{G} \gamma_{\theta, \sigma}$, we have

$$
\operatorname{Ind}_{N}^{G} \gamma_{\theta, \sigma}=\int_{G \cdot q^{-1}(\theta, \sigma) / G}^{\oplus} \pi_{(\varphi, \tau)}
$$


Similarly, Theorem 2 is asserting (in Kirillov's terminology) that

$$
\operatorname{Ind}_{H}^{G} \nu_{\psi}=\int_{G \cdot q^{-1}(\psi) / G}^{\oplus} \pi_{(\psi, \tau)},
$$

where now $q^{-1}(\psi)=\left\{(\varphi, \tau) \in \mathcal{R}_{\mathfrak{g}}:\left.\varphi\right|_{\mathfrak{h}}=\psi\right\}$. Thus we see that, after applying the induction hypothesis, equality of spectrum amounts to the equality

$$
G \cdot q_{\mathfrak{g}, \mathfrak{h}}^{-1}(\psi)=G \cdot q_{\mathfrak{g}, \mathfrak{n}}^{-1}\left(N \cdot q_{\mathfrak{n}, \mathfrak{h}}^{-1}(\psi)\right) .
$$

This is essentially obvious, once we check that for $\varphi \in \mathfrak{g}^{*},\left.\varphi\right|_{\mathfrak{n}}=\theta,\left.\varphi\right|_{\mathfrak{h}}=\psi$, we have

$$
\begin{aligned}
& \mathcal{X}_{\sigma}(\varphi) \neq \emptyset, \quad \forall \sigma \in \mathcal{X}(\theta) \\
& \mathcal{X}(\varphi)=\bigcup_{\sigma \in \mathcal{X}(\theta)} \mathcal{X}_{\sigma}(\varphi) .
\end{aligned}
$$

These are both immediate consequences of the fact that in every one of the four cases in $\S 2$, we have

$$
G_{\psi}^{0} \cap N_{\varphi}=N_{\varphi}^{0}
$$

Multiplicity. This is the heart of argument. Not unexpectedly, the proof is long and complicated. We have already observed that the multiplicity on the right side of $(3.2)$ is

$$
n_{\varphi}^{\psi}=\# H \text {-orbits on } G \cdot \varphi \cap p^{-1}(H \cdot \psi) .
$$

Of course we are using the nilpotence of $H$ here. Without it we would have to take into account $\mathcal{X}(\varphi)$, and we might not have uniform multiplicity over the space $\mathcal{X}(\varphi)$ as we do here. Now to relate $n_{\varphi}^{\psi}$ to the left side of (3.2) we need some auxiliary results. The first is the analog of [7, Proposition 1.7], [8, Lemma 4.2]. As in [8] a generic set here means one whose interior has Lebesgue null complement.

Lemma 3.1. Let $H \subset N \triangleleft G$ be simply connected solvable Lie groups. Assume $H$ is nilpotent, $G$ and $N$ are type I and $\mathfrak{n}^{*} / G$ is countably separated. Fix $\psi \in \mathfrak{h}^{*}$. Then generically on $p_{\mathfrak{n}, \mathfrak{h}}^{-1}(H \cdot \psi)$ we have

$$
G \cdot \theta \cap p_{\mathfrak{n}, \mathfrak{h}}^{-1}(H \cdot \psi) \text { has the same dimension as } \mathfrak{g} \cdot \theta \cap p_{\mathfrak{n}, \mathfrak{h}}^{-1}(\mathfrak{h} \cdot \psi)
$$

Proof. The idea of the proof is the same as [8, Lemma 4.2]. The action of $G$ on $\mathfrak{n}^{*}$ is smooth but since it is not exponential solvable, eigenvalues on the unit circle may occur. Nevertheless there is still a locally smooth cross-section - the parameter set for the cross-section may collapse from 'flat' to 'circular' in certain 
places. More precisely, there exists an open $G$-invariant co-null set $\mathcal{U} \subset \mathfrak{n}^{*}$ such that $\forall \theta \in \mathcal{U}, \exists G$-invariant $\mathcal{O}_{\theta}$ such that:

$$
\mathcal{O}_{\theta} \cap p_{\mathfrak{n}, \mathfrak{h}}^{-1}(H \cdot \psi) \text { is open, co-null in } p_{\mathfrak{n}, \mathfrak{h}}^{-1}(H \cdot \psi)
$$

and a decomposition $\mathcal{O}_{\theta}=\bigcup_{i} \mathcal{O}_{\theta}^{i}$ into $G$-invariant open sets; and non-singular, bi-rational maps

$$
\Phi_{i}: \mathcal{O}_{\theta}^{i} \longrightarrow \Sigma \times V^{i}
$$

where $\Sigma$ is an open ball in Euclidean space, $V^{i}=\mathbf{R}^{n_{i}} \times \mathbf{T}^{m_{i}}, n_{i}+m_{i}$ is independent of $i$, and

$$
\begin{gathered}
\Phi_{i}^{-1}\left\{\left(\sigma, v_{i}\right): v_{i} \in V^{i}\right\} \text { constitutes a } G \text {-orbit, } \forall \sigma \in \Sigma \\
\Phi_{i}^{-1}\left(\sigma, v_{i}\right), \Phi_{j}^{-1}\left(\sigma^{\prime}, v_{j}\right) \text { lie in the same orbit } \Longleftrightarrow i=j \text { and } \sigma=\sigma^{\prime} .
\end{gathered}
$$

Using this set up, the remainder of the proof is virtually identical to that of $[8$, Lemma 4.2] (except we replace $\mathfrak{h}^{\perp}$ by $p^{-1}(\mathfrak{h} \cdot \psi)=\mathfrak{h}_{\psi}^{\perp}$ as in [7, Prop. 1.7]).

Lemma 3.1 is crucial for evaluating multiplicities. For example, on the right side of $(3.2)$ : if generically on $p_{\mathfrak{g}, \mathfrak{h}}^{-1}(\mathfrak{h} \cdot \psi)$ we have

$$
\operatorname{dim} \mathfrak{h} \cdot \varphi<\operatorname{dim} \mathfrak{g} \cdot \varphi \cap p_{\mathfrak{g}, \mathfrak{h}}^{-1}(\mathfrak{h} \cdot \psi)
$$

then the multiplicity is uniformly infinite. In the complementary situation the multiplicities may be finite or infinite, and our argument must deal with either eventuality.

Now we set up a partition according to the four possibilities in Theorem 1. For every $\varphi \in \mathfrak{g}^{*}$, set $\theta=\varphi \mid \mathfrak{n}$. Then we denote

$$
\begin{aligned}
& \mathfrak{g}_{1}^{*}=\left\{\varphi \in \mathfrak{g}^{*}: G_{\theta}=N_{\theta}\right\} \\
& \mathfrak{g}_{2}^{*}=\left\{\varphi \in \mathfrak{g}^{*}: G_{\theta} / N_{\theta} \cong \mathbf{Z}\right\} \\
& \mathfrak{g}_{3}^{*}=\left\{\varphi \in \mathfrak{g}^{*}: G_{\theta} / N_{\theta} \cong \mathbf{R}, G_{\varphi}=G_{\theta}\right\} \\
& \mathfrak{g}_{4}^{*}=\left\{\varphi \in \mathfrak{g}^{*}: G_{\theta} / N_{\theta} \cong \mathbf{R}, G_{\varphi} \neq G_{\theta}\right\}
\end{aligned}
$$

Clearly $\mathfrak{g}^{*}=\bigcup_{j=1}^{4} \mathfrak{g}_{j}^{*}$ is a $G$-invariant partition. Similarly if we set $\mathfrak{n}_{j}^{*}=p_{\mathfrak{g}, \mathfrak{n}}\left(\mathfrak{g}_{j}^{*}\right)$, $1 \leq j \leq 4$, we obtain a $G$-invariant partition of $\mathfrak{n}^{*}$. Now it is a simple consequence of Theorem 1 that the equivalence of two induced representations $\operatorname{Ind}_{N}^{G} \gamma_{\theta_{j}, \sigma_{j}}, j=1,2$, can occur only if $\theta_{1}$ and $\theta_{2}$ are in the same cell $\mathfrak{n}_{j}^{*}$. Moreover it is also clear that two direct integrals of the form

$$
\int_{\mathcal{X}\left(\theta_{j}\right)}^{\oplus} \operatorname{Ind}_{N}^{G} \gamma_{\theta_{j}, \sigma_{j}} d \sigma_{j}, \quad j=1,2,
$$


can be equivalent only if $\theta_{1}$ and $\theta_{2}$ are in the same $G$-orbit. These observations, together with formulas (S1), (S2), show that the multiplicity on the left side of (3.2) equals

$$
\# H \text {-orbits on } G \cdot \theta \cap p_{\mathfrak{n}, \mathfrak{h}}^{-1}(H \cdot \psi) \text {. }
$$

We shall use Lemma 3.1 to know that the dimension of $G \cdot \theta \cap p_{\mathfrak{n}, \mathfrak{h}}^{-1}(\mathfrak{h} \cdot \varphi)$ agrees with that of $\mathfrak{g} \cdot \theta \cap p_{\mathfrak{n}, \mathfrak{h}}^{-1}(\mathfrak{h} \cdot \varphi)$.

Now, as is usual in these arguments ([7] [8]), we have to partition $p_{\mathfrak{g}, \mathfrak{h}}^{-1}(H \cdot \psi)$ according to the generic dimensions of the $G$ and $H$ orbits passing through it. We distinguish two mutually exclusive cases

$$
\begin{aligned}
& \operatorname{dim} G \cdot \varphi \cap p_{\mathfrak{g}, \mathfrak{h}}^{-1}(H \cdot \psi)>\operatorname{dim} H \cdot \varphi \\
& \operatorname{dim} G \cdot \varphi \cap p_{\mathfrak{g}, \mathfrak{h}}^{-1}(H \cdot \psi)=\operatorname{dim} H \cdot \varphi
\end{aligned}
$$

By Lemma 3.1, these are equivalent to

$$
\begin{aligned}
& \operatorname{dim} \mathfrak{g} \cdot \varphi \cap p_{\mathfrak{g}, \mathfrak{h}}^{-1}(\mathfrak{h} \cdot \psi)>\operatorname{dim} \mathfrak{h} \cdot \varphi \\
& \operatorname{dim} \mathfrak{g} \cdot \varphi \cap p_{\mathfrak{g}, \mathfrak{h}}^{-1}(\mathfrak{h} \cdot \psi)=\operatorname{dim} \mathfrak{h} \cdot \varphi
\end{aligned}
$$

Furthermore (see Lemma 3.2) below, these are equivalent to

$$
\begin{aligned}
\operatorname{dim} \mathfrak{g} \cdot \varphi & >2 \operatorname{dim} \mathfrak{h} \cdot \varphi-\operatorname{dim} \mathfrak{h} \cdot \psi \\
\operatorname{dim} \mathfrak{g} \cdot \varphi & =2 \operatorname{dim} \mathfrak{h} \cdot \varphi-\operatorname{dim} \mathfrak{h} \cdot \psi
\end{aligned}
$$

REMARK. We shall prove equal multiplicity by considering the eight possible cases according to $1 \leq j \leq 4$, and we are in situation (a) or (b). (Some of the cases will coalesce.) It may be that in any example the number of cases that can occur is limited - perhaps even to one. Unfortunately I do not have any such general result. So I shall treat each case independently. Here is the promised

Lemma 3.2. For any $\varphi \in \mathfrak{g}^{*}, \theta=\left.\varphi\right|_{\mathfrak{n}}, \psi=\left.\varphi\right|_{\mathfrak{h}}$, we have

$$
\mathfrak{g} \cdot \theta=\mathfrak{n}_{\varphi}^{\perp}(\mathfrak{n})
$$
$\operatorname{dim} \mathfrak{g} \cdot \theta \cap p_{\mathfrak{n}, \mathfrak{h}}^{-1}(\mathfrak{h} \cdot \psi)=\operatorname{dim} \mathfrak{g} \cdot \theta-\operatorname{dim} \mathfrak{h} \cdot \varphi+\operatorname{dim} \mathfrak{h} \cdot \psi$ $\operatorname{dim} \mathfrak{g} \cdot \varphi \cap p_{\mathfrak{g}, \mathfrak{h}}^{-1}(\mathfrak{h} \cdot \psi)=\operatorname{dim} \mathfrak{g} \cdot \varphi-\operatorname{dim} \mathfrak{h} \cdot \varphi+\operatorname{dim} \mathfrak{h} \cdot \psi$

Proof. (i) We begin by noting that

$$
\mathfrak{g} \cdot \theta\left(\mathfrak{n}_{\varphi}\right)=\theta\left[\mathfrak{g}, \mathfrak{n}_{\varphi}\right] \subset \varphi\left[\mathfrak{g}, \mathfrak{g}_{\varphi}\right]=\{0\}
$$


But $\operatorname{dim} \mathfrak{g} \cdot \theta=\operatorname{dim} \mathfrak{g} / \mathfrak{g}_{\theta}$ and $\operatorname{dim} \mathfrak{n}_{\varphi}^{\perp}(\mathfrak{n})=\operatorname{dim} \mathfrak{n} / \mathfrak{n}_{\varphi}$. However a review of Theorem 1 reveals that, in every one of the four cases, we have $\operatorname{dim} \mathfrak{g}_{\theta}=\operatorname{dim} \mathfrak{n}_{\varphi}+1$. The conclusion $\mathfrak{g} \cdot \theta=\mathfrak{n}_{\varphi}^{\perp}(\mathfrak{n})$ follows immediately.

(ii) Using part (i) we compute

$$
\begin{aligned}
& \operatorname{dim} \mathfrak{g} \cdot \theta \cap p_{\mathfrak{n}, \mathfrak{h}}^{-1}(\mathfrak{h} \cdot \psi)=\operatorname{dim} \mathfrak{g} \cdot \theta \cap \mathfrak{h}_{\psi}^{\perp}=\operatorname{dim} \mathfrak{n}_{\varphi}^{\perp} \cap \mathfrak{h}_{\psi}^{\perp} \\
= & \operatorname{dim}\left(\mathfrak{n}_{\varphi}+\mathfrak{h}_{\psi}\right)^{\perp}=\operatorname{dim} \mathfrak{n} /\left(\mathfrak{n}_{\varphi}+\mathfrak{h}_{\psi}\right) \\
= & \operatorname{dim} \mathfrak{n}-\operatorname{dim} \mathfrak{n}_{\varphi}-\operatorname{dim} \mathfrak{h}_{\psi}+\operatorname{dim}\left(\mathfrak{n}_{\varphi} \cap \mathfrak{h}_{\psi}\right) \\
= & \operatorname{dim} \mathfrak{n}-\operatorname{dim} \mathfrak{n}_{\varphi}-\operatorname{dim} \mathfrak{h}_{\psi}+\operatorname{dim} \mathfrak{h}_{\varphi} \\
= & \operatorname{dim} \mathfrak{n} / \mathfrak{n}_{\varphi}-\left(\operatorname{dim} \mathfrak{h}-\operatorname{dim} \mathfrak{h}_{\varphi}\right)-\left(\operatorname{dim} \mathfrak{h}-\operatorname{dim} \mathfrak{h}_{\psi}\right) \\
= & \operatorname{dim} \mathfrak{g} \cdot \theta-\operatorname{dim} \mathfrak{h} \cdot \varphi+\operatorname{dim} \mathfrak{h} \cdot \psi .
\end{aligned}
$$

(iii) This is similar to (ii).

$$
\begin{aligned}
& \operatorname{dim} \mathfrak{g} \cdot \varphi \cap p_{\mathfrak{g}, \mathfrak{h}}^{-1}(\mathfrak{h} \cdot \psi)=\operatorname{dim} \mathfrak{g}_{\varphi}^{\perp} \cap \mathfrak{h}_{\psi}^{\perp} \\
= & \operatorname{dim} \mathfrak{g}-\operatorname{dim} \mathfrak{g}_{\varphi}-\operatorname{dim} \mathfrak{h}_{\psi}+\operatorname{dim} \mathfrak{h}_{\varphi} \\
= & \operatorname{dim} \mathfrak{g} \cdot \varphi-\operatorname{dim} \mathfrak{h} \cdot \psi+\operatorname{dim} \mathfrak{h} \cdot \psi .
\end{aligned}
$$

We turn at last to the proof of equal multiplicity in formula (3.2) for the eight cases outlined above. In each case we will prove equal multiplicity by showing: either that both sides have uniform infinite multiplicity, or that

$$
\# H \text {-orbits on } G \cdot \varphi \cap p_{\mathfrak{g}, \mathfrak{h}}^{-1}(H \cdot \psi)=\# H \text {-orbits on } G \cdot \theta \cap p_{\mathfrak{n}, \mathfrak{h}}^{-1}(H \cdot \psi) \text {. }
$$

During the argument we keep in mind the following facts: (1) on the varieties in question, we have generically that

$$
\begin{aligned}
& \operatorname{dim} G \cdot \varphi \cap p_{\mathfrak{g}, \mathfrak{h}}^{-1}(H \cdot \psi)=\operatorname{dim} \mathfrak{g} \cdot \varphi \cap p_{\mathfrak{g}, \mathfrak{h}}^{-1}(h \cdot \psi) \\
& \operatorname{dim} G \cdot \theta \cap p_{\mathfrak{n}, \mathfrak{h}}^{-1}(H \cdot \psi)=\operatorname{dim} \mathfrak{g} \cdot \theta \cap p_{\mathfrak{n}, \mathfrak{h}}^{-1}(\mathfrak{h} \cdot \psi) ;
\end{aligned}
$$

(2) equations (3.3)-(3.5); (3) Lemmas 3.1 and 3.2; and (4) that all co-adjoint orbits are even-dimensional.

(a) We assume here that generically on $p_{\mathfrak{g}, \mathfrak{h}}^{-1}(H \cdot \psi)$ we have

$$
\operatorname{dim} \mathfrak{g} \cdot \varphi>2 \operatorname{dim} \mathfrak{h} \cdot \varphi-\operatorname{dim} \mathfrak{h} \cdot \psi .
$$

Then the multiplicity is uniformly infinite on the right side of (3.2). To show uniform infinite multiplicity on the left side of $(3.2)$, it is enough to prove

$$
\operatorname{dim} \mathfrak{g} \cdot \theta \cap p_{\mathfrak{n}, \mathfrak{h}}^{-1}(\mathfrak{h} \cdot \psi)>\operatorname{dim} \mathfrak{h} \cdot \theta
$$


By Lemma 3.2 (ii), that is tantamount to proving

$$
\operatorname{dim} \mathfrak{g} \cdot \theta-\operatorname{dim} \mathfrak{h} \cdot \varphi+\operatorname{dim} \mathfrak{h} \cdot \psi>\operatorname{dim} \mathfrak{h} \cdot \theta .
$$

But for that it suffices to show

$$
\operatorname{dim} \mathfrak{g} \cdot \theta \geq \operatorname{dim} \mathfrak{g} \cdot \varphi-1
$$

Indeed, given (3.8), and using the evenness of both sides of (3.7), we have

$$
\begin{gathered}
\operatorname{dim} \mathfrak{g} \cdot \theta \geq \operatorname{dim} \mathfrak{g} \cdot \varphi-1>2 \operatorname{dim} \mathfrak{h} \cdot \varphi-\operatorname{dim} \mathfrak{h} \cdot \psi \\
\geq \operatorname{dim} \mathfrak{h} \cdot \varphi+\operatorname{dim} \mathfrak{h} \cdot \theta-\operatorname{dim} \mathfrak{h} \cdot \psi .
\end{gathered}
$$

But (3.8) is a triviality. It just says that $\operatorname{dim} \mathfrak{g}_{\varphi} \geq \operatorname{dim} \mathfrak{g}_{\theta}-1-$ which is completely evident from the four cases. We observe at this point that no assumption on $G_{\theta}$ or $N_{\theta}$ was used in the above argument - the exact same argument works in all four cases of Theorem 1. That is, inequality (3.6) forces infinite multiplicity on both sides of (3.2). Therefore we can restrict ourselves to case (b) in all of what follows.

(b) We assume $\operatorname{dim} \mathfrak{g} \cdot \varphi=2 \operatorname{dim} \mathfrak{h} \cdot \varphi-\operatorname{dim} \mathfrak{h} \cdot \psi$ generically on $p_{\mathfrak{g}, \mathfrak{h}}^{-1}(\mathfrak{h} \cdot \psi)$. Unlike case (a) we must treat the four cases of Theorem 1 separately. In (i)-(iii) we shall demonstrate the bijection asserted in (3.6). In (iv) we shall once again prove both sides have infinite multiplicity.

(i) In this case $G_{\theta}=N_{\theta}$. We prove that the restriction map

$$
G \cdot \varphi \cap p_{\mathfrak{g} \cdot \mathfrak{h}}^{-1}(H \cdot \psi) \longrightarrow G \cdot \theta \cap p_{\mathfrak{n} \cdot \mathfrak{h}}^{-1}(H \cdot \psi)
$$

sets up a bijection of $H$-orbits. It is clearly a surjective $H$-equivariant map. We must show that if $\varphi \in p^{-1}(H \cdot \psi)$ and $g \cdot \varphi$ have the property that $h \cdot \theta=g \cdot \theta$, then $\varphi$ and $g \cdot \varphi$ lie in the same $H$-orbit. We start by observing

$$
\begin{gathered}
2 \operatorname{dim} \mathfrak{h} \cdot \theta-\operatorname{dim} \mathfrak{h} \cdot \psi \leq \operatorname{dim} \mathfrak{n} \cdot \theta=\operatorname{dim} \mathfrak{n} / \mathfrak{n}_{\theta}=\operatorname{dim} \mathfrak{g} / \mathfrak{g}_{\theta}-1 \\
=\operatorname{dim} \mathfrak{g} / \mathfrak{g}_{\varphi}-2=2(\operatorname{dim} \mathfrak{h} \cdot \varphi-1)-\operatorname{dim} \mathfrak{h} \cdot \psi
\end{gathered}
$$

That is

$$
\operatorname{dim} \mathfrak{h} \cdot \theta \leq \operatorname{dim} \mathfrak{h} \cdot \varphi-1
$$

Strict inequality is impossible, therefore

$$
\operatorname{dim} \mathfrak{h} \cdot \theta=\operatorname{dim} \mathfrak{h} \cdot \varphi-1 .
$$

So $H_{\theta}^{0} \cdot \varphi$ is an open connected subset of $\varphi+\mathfrak{n}^{\perp}=N_{\theta} \cdot \varphi$. This is true for any $\varphi=p^{-1}(\theta)$, and the conclusion is therefore that

$$
H_{\theta} \cdot \varphi=N_{\theta} \cdot \varphi=\varphi+\mathfrak{n}^{\perp} .
$$


Thus if $h \cdot \theta=g \cdot \theta$, we have $h^{-1} g \in G_{\theta}=N_{\theta} \Rightarrow h^{-1} g \cdot \varphi=h_{\theta} \cdot \varphi$ for some $h_{\theta} \in H_{\theta}$. That is $g \cdot \varphi=h h_{\theta} \cdot \varphi$, exactly what we had to prove.

(ii) In this case $G_{\theta}^{0}=N_{\theta}^{0}$ and $N_{\theta}^{0} \cdot \varphi=\varphi+\mathfrak{n}^{\perp}=H_{\theta} \cdot \varphi$, by the same argument as in (i). The rest of the reasoning from that case is still valid.

(iii) In this case the mapping

$$
G \cdot \varphi \cap p_{\mathfrak{g} \cdot \mathfrak{h}}^{-1}(H \cdot \psi) \longrightarrow G \cdot \theta \cap p_{\mathfrak{n} \cdot \mathfrak{h}}^{-1}(H \cdot \psi)
$$

is actually a bijection itself - a fortiori a bijection of $H$-orbits. To show that it suffices to verify that if two functionals $\varphi, g \cdot \varphi$ restrict to the same functional on $\mathfrak{n}$, then they coincide. But $\theta=\left.\varphi\right|_{\mathfrak{n}}=\left.g \cdot \varphi\right|_{\mathfrak{n}} \Rightarrow g \in G_{\theta}$. Since in this case $G_{\theta}=G_{\varphi}$, the assertion is clear.

We come to the last and most interesting case.

(iv) In fact there is no bijection of $H$-orbits here (see Example 3.3 ). We demonstrate equality of multiplicity by showing that both sides of (3.2) are of infinite multiplicity. We prove it on the left side by showing that $\int_{\mathcal{X}(\theta)}^{\oplus} \operatorname{Ind}_{N}^{G} \gamma_{\theta, \sigma} d \sigma$ is of uniform infinite multiplicity whenever $G_{\theta} / N_{\theta} \cong \mathbf{R}$ and $G_{\theta} \neq\left(G_{\theta}\right)_{\sigma}$. Actually it is possible that $\left(G_{\theta}\right)_{\sigma}$ varies with $\sigma$. That is not important. What is important is - as we saw in the proof of case (iv) in Theorem 1 - that $\left(G_{\theta}\right)_{\sigma} / N_{\theta} \cong \mathbf{Z}$, so that $\left(G_{\theta}\right) /\left(G_{\theta}\right)_{\sigma} \cong \mathbf{T}$. Consider the action of $G_{\theta}$ on $\mathcal{X}(\theta)$. Let $\mathcal{C}$ be a Borel crosssection for the action. In the terminology of Figure $4, \mathcal{C}$ will be an $r$-torus $(r \geq 0)$ with its Lebesgue measure class. Then we compute

$$
\begin{aligned}
\int_{\mathcal{X}(\theta)}^{\oplus} \operatorname{Ind}_{N}^{G} \gamma_{\theta, \sigma} & =\int_{\mathcal{C}}^{\oplus} \int_{G_{\theta} /\left(G_{\theta}\right)_{\sigma}}^{\oplus} \operatorname{Ind}_{N}^{G} \gamma_{\theta, g \cdot \sigma} d \dot{\sigma} d \dot{g} \\
& =\int_{\mathcal{C}}^{\oplus} \int_{G_{\theta} /\left(G_{\theta}\right)_{\sigma}}^{\oplus} \operatorname{Ind}_{N}^{G} g \cdot \gamma_{\theta, \sigma} d \dot{\sigma} d \dot{g} \\
& =\int_{\mathcal{C}}^{\oplus} \int_{G_{\theta} /\left(G_{\theta}\right)_{\sigma}}^{\oplus} g \cdot \operatorname{Ind}_{N}^{G} \gamma_{\theta, \sigma} d \dot{\sigma} d \dot{g} \\
& =\infty \int_{\mathcal{C}}^{\oplus} \operatorname{Ind}_{N}^{G} \gamma_{\theta, \sigma} d \dot{\sigma} .
\end{aligned}
$$

Now we deal with the right side of (3.2). We consider the usual map

$$
G \cdot \varphi \cap p_{\mathfrak{g} \cdot \mathfrak{h}}^{-1}(H \cdot \psi) \longrightarrow G \cdot \theta \cap p_{\mathfrak{n} \cdot \mathfrak{h}}^{-1}(H \cdot \psi),
$$

an $H$-equivariant surjection. In this case we have $G_{\theta}=G_{\varphi}^{0} N_{\theta}$ and so

$$
G_{\theta} \cdot \varphi=\varphi+s_{0} \mathbf{Z} \alpha \subset p^{-1}(H \cdot \psi)
$$


(see the proof of (iv) in Section 2). These functionals are actually in distinct $N$ orbits. For otherwise

$$
g_{\theta} \cdot \varphi=\varphi+n s_{0} \alpha=h \cdot \varphi, \quad \text { some } \quad h \in H .
$$

This implies $h^{-1} g \in G_{\theta}$, i.e. $h \in H \cap G_{\theta}=H_{\theta}$. But the assumption $H \subset$ Nilrad $G$ forces $H_{\theta}$ to be connected. Thus $H_{\theta} \cdot \varphi$ connected and contained in $\varphi+s_{0} \mathbf{Z} \alpha$, implies $H_{\theta}=H_{\varphi}$. The functionals $\varphi+s_{0} \mathbf{Z} \alpha$, therefore, lie in distinct $H$-orbits. Hence

$$
\#\left[G \cdot \varphi \cap p_{\mathfrak{g} \cdot \mathfrak{h}}^{-1}(H \cdot \psi)\right] / H=+\infty,
$$

and so the right side of (3.2) also has infinite multiplicity.

This completes the proof of equal multiplicity. It remains only to prove the equivalence of the

Spectral Measures. We begin with the observation that

$$
p: p_{\mathfrak{g}, \mathfrak{h}}^{-1}(H \cdot \psi) \longrightarrow p_{\mathfrak{n}, \mathfrak{h}}^{-1}(H \cdot \psi)
$$

clearly maps the canonical measure class of the former to that of the latter. After factoring by $H$, it is also clear that $p$ takes the measure class of $d \dot{\varphi}$ to that of $d \dot{\theta}$ in (3.2). Now we pass to a case-by-case analysis.

(i) $G_{\theta}=N_{\theta}$. We exhibit a Borel isomorphism $\mathcal{X}(\theta) \rightarrow \mathcal{X}(\varphi)$ which converts $d \sigma$ to $d \tau$. In fact since $N_{\varphi}=G_{\varphi}$, the map is simply $\sigma \rightarrow \tau=\left.\sigma\right|_{N_{\varphi}}$. We already observed in $\S 2$ that the map is well-defined, and it is clearly Borel. That it is an isomorphism follows because $N_{\theta}=N_{\theta}^{0} N_{\varphi}$ and $N_{\theta}^{0} \cap N_{\varphi}=N_{\varphi}^{0}$. (see Theorem 1 case (i) in $\S 2)$.

(ii) As in (i), $p$ set up an isomorphism of the measure classes of $d \dot{\varphi}$ and $d \dot{\theta}$, but this time $\operatorname{Ind}_{N}^{G} \gamma_{\theta, \sigma}$ is not irreducible

$$
\operatorname{Ind}_{N}^{G} \gamma_{\theta, \sigma}=\int_{\mathcal{X}_{\sigma}(\varphi)}^{\oplus} \pi_{\varphi, \tau} d \tau .
$$

The equality of measures follows from the diagram

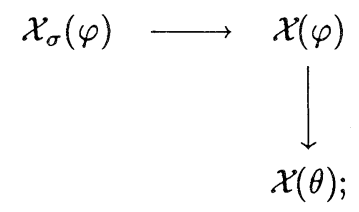

that is $\mathcal{X}(\varphi)$ is a fiber space over $\mathcal{X}(\theta)$ with fiber $\mathcal{X}_{\sigma}(\varphi)$. This follows easily from Figure 2 and the fact $G_{\theta}=G_{\varphi} N_{\theta}^{0}$ in case (ii). The fiber map is $\tau \rightarrow \sigma$, where $\tau$ extends uniquely to $G_{\theta}$ by $g_{\varphi} n_{\theta}^{0} \rightarrow \tau\left(g_{\varphi}\right) \chi_{\theta}\left(n_{\theta}^{0}\right)$ and $\sigma=\left.\tau\right|_{N_{\theta}}$. The map is clearly surjective and the fiber is precisely $\mathcal{X}_{\sigma}(\varphi)$. 
Note that: $\mathcal{X}(\theta)$ is an $r$-torus, $\mathcal{X}(\varphi)$ is an $(r+1)$-torus and $\mathcal{X}_{\sigma}(\varphi)$ is a 1-torus. All measure classes - base and fiber - are Lebesgue.

(iii) In this case the tori $\mathcal{X}(\varphi)$ and $\mathcal{X}(\theta)$ are in Borel correspondence, as follows from Figure 3. It is the outside integrals in (3.2) which are fibered. Situations analogous to this case have been handled in $[7, \S 2]$ and $[8, \S 4]$, and nothing fundamentally new occurs here. Namely, the map $\varphi+s \alpha \rightarrow \theta$ gives the fiber structure

$$
\mathbf{R} \longrightarrow \begin{gathered}
p_{\mathfrak{g} \cdot \mathfrak{h}}^{-1}(H \cdot \psi) / H \\
\downarrow \\
p_{\mathfrak{n} \cdot \mathfrak{h}}^{-1}(H \cdot \psi) / H ;
\end{gathered}
$$

and since $\operatorname{Ind}_{N}^{G} \gamma_{\theta, \sigma}=\int_{\mathbf{R}}^{\oplus} \pi_{\varphi+s \alpha, \tau_{S}} d s$, the measures on either side of (3.1) agree.

(iv) Actually this case is virtually identical to (iii). Once again the tori $\mathcal{X}(\varphi)$ and $\mathcal{X}(\theta)$ are in Borel correspondence - it is only that the fiber is a circle instead of a line. The fiber picture becomes

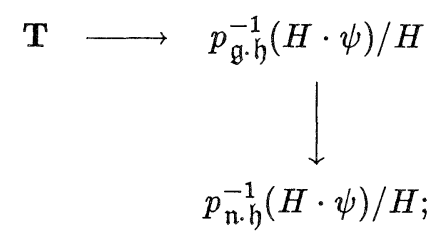

This finishes the proof of equality of spectral measures in (3.2), and with it the proof of Theorem 2. We conclude the paper with an example to illustrate the unusual features of case (iv).

EXAMPLE 3.3.

$$
\begin{aligned}
& \mathfrak{g}=\operatorname{sp}\{A, X, Y, U, V\} ;[A, X]=Y,[A, Y]=-X,[A, U]=V \\
& \mathfrak{n}=\operatorname{sp}\{A, X, Y, V\}, \mathfrak{h}=\operatorname{sp}\{X, Y, V\} \\
& \psi=X^{*}+V^{*} \in \mathfrak{h}^{*}, \operatorname{Ind}_{H}^{G} \nu_{\psi}=\operatorname{Ind}_{H}^{G} \chi_{\psi} \\
& g=\exp a A \exp x X \exp y Y \exp u U \exp v V \\
& \varphi=\alpha A^{*}+\xi X^{*}+\eta Y^{*}+\mu U^{*}+\nu V^{*}=\varphi(\alpha, \xi, \eta, \mu, \nu) \in \mathfrak{g}^{*} \\
& g^{-1} \cdot \varphi=\varphi(\alpha-u \nu+\xi(y \cos a+x \sin a)+\eta(y \sin a-x \cos a), \\
& \quad \xi \cos a+\eta \sin a,-\xi \sin a+\eta \cos a, \mu+a \nu, \nu)
\end{aligned}
$$




$$
\begin{aligned}
& p_{\mathfrak{g} \cdot \mathfrak{h}}^{-1}(H \cdot \psi)=p^{-1}(\psi)=\{\varphi(\alpha, 1,0, \mu, 1): \alpha, \mu \in \mathbf{R}\} \\
& \varphi \in p_{\mathfrak{g}, \mathfrak{h}}^{-1}(\psi): G \cdot \varphi=\{\varphi(\alpha-u+(y \cos a+x \sin a), \\
& \quad \cos a,-\sin a, \mu+a, 1)\} \\
& G_{\varphi}=\{g=\exp x X \exp \lambda(Y+U) \exp v V: x, \lambda, v \in \mathbf{R}\}, \quad \text { connected } \\
& \pi_{\varphi}=\operatorname{Ind}_{M}^{G} \chi_{\varphi}, \mathfrak{m}=\operatorname{sp}\{X, Y, U, V\} .
\end{aligned}
$$

Cross-section for $p^{-1}(\psi)$ is $\mathcal{C}=\{\varphi(0,1,0, \mu, 1): 0 \leq \mu<2 \pi\}$

$$
\begin{gathered}
\varphi \in \mathcal{C}: G \cdot \varphi \cap p^{-1}(H \cdot \psi)=\{\varphi(\alpha, 1,0, \mu+2 \pi n, 1): \alpha \in \mathbf{R}, n \in \mathbf{Z}\} \\
H \cdot \varphi=\{\varphi(y, 1,0, \mu, 1): y \in \mathbf{R}\} .
\end{gathered}
$$

$H$ has infinitely many open orbits on $G \cdot \varphi \cap p^{-1}(H \cdot \psi)$ and

$$
\operatorname{Ind}_{H}^{G} \chi_{\psi}=\infty \int_{[0,2 \pi]}^{\oplus} \operatorname{Ind}_{M}^{G} \chi_{1,0, \mu, 1} d \mu .
$$

Now redo the calculation by passing through $N$.

$$
\begin{gathered}
\theta=\theta(\alpha, \xi, \eta, \nu)=a A^{*}+\xi X^{*}+\eta Y^{*}+\nu V^{*} \in \mathfrak{n}^{*} \\
n^{-1} \cdot \theta=\theta(\alpha+\xi(y \cos a+x \sin a)+\eta(y \sin a-x \cos a), \\
\xi \cos a+\eta \sin a,-\xi \sin a+\eta \cos a, \nu) \\
p_{\mathfrak{n}, \mathfrak{h}}^{-1}(H \cdot \psi)=\{\theta(\alpha, 1,0,1): \alpha \in \mathbf{R}\} \\
\theta \in p_{\mathfrak{n}, \mathfrak{h}}^{-1}(H \cdot \psi): N \cdot \theta=\{\theta(\alpha+y \cos a+x \sin a, \cos a,-\sin a, 1)\} \\
N_{\theta}=C N_{\theta}^{0}, N_{\theta}^{0}=\{n=\exp x X \exp v V: x, v \in \mathbf{R}\}, C=\{\exp 2 \pi n A: n \in \mathbf{Z}\} \\
p_{\mathfrak{n}, \mathfrak{h}}^{-1}(\psi) \text { meets only one orbit, namely } \theta_{0}=\theta(0,1,0,1) \\
\operatorname{Ind}_{H}^{N} \chi_{\psi}=\int_{\widehat{C}}^{\oplus} \gamma_{\theta_{0}, \sigma} d \sigma .
\end{gathered}
$$

Next $g^{-1} \cdot \theta_{0}=\theta(-u+y \cos a+x \sin a, \cos a,-\sin a, 1)$

$$
G_{\theta_{0}}=C G_{\theta_{0}}^{0}, G_{\theta_{0}}^{0}=\{g=\exp x X \exp \lambda(Y+U) \exp \nu V\}, G_{\theta} / N_{\theta} \cong \mathbf{R} .
$$

But $\left(G_{\theta_{0}}\right)_{\sigma}=C\{\exp x X \exp \lambda(Y+U) \exp v V: \lambda \in \mathbf{Z}\} \neq G_{\theta}$.

Clearly $\mathcal{X}(\theta)$ is a homogeneous $G_{\theta}$-space, so

$$
\int^{\oplus} \gamma_{\theta_{0}, \sigma} d \sigma=\int_{G / G_{\gamma_{\theta_{0}, 1}}}^{\oplus} g \cdot \gamma_{\theta_{0}, 1} d \dot{g} .
$$


Therefore

$$
\begin{aligned}
\operatorname{Ind}_{H}^{G} \chi_{\psi} & =\operatorname{Ind}_{N}^{G} \operatorname{Ind}_{H}^{N} \chi_{\psi}=\operatorname{Ind}_{N}^{G} \int^{\oplus} \gamma_{\theta_{0}, \sigma} d \sigma=\operatorname{Ind}_{N}^{G} \int_{G / G_{\gamma_{0}, 1}}^{\oplus} g \cdot \gamma_{\theta_{0}, 1} d \dot{g} \\
& =\int_{G / G_{\gamma_{\theta_{0}}, 1}}^{\oplus} \operatorname{Ind}_{N}^{G} g \cdot \gamma_{\theta_{0}, 1} d \dot{g}=\infty \operatorname{Ind}_{N}^{G} \gamma_{\theta_{0}, 1} .
\end{aligned}
$$

The representation $\operatorname{Ind}_{N}^{G} \gamma_{\theta_{0}, 1}$ is, by [5], multiplicity-free with spectrum consisting of the representations $\pi_{\varphi}$ corresponding to those $\varphi$ lying over $\theta_{0}$. This is precisely the spectrum of $(3.9)$.

\title{
References
}

[1] L. Auslander and B. Kostant, Polarization and unitary representations of solvable Lie groups, Invent. Math., 14 (1971), 255-354.

[2] P. Bernat, et al., Représentations des groupes de Lie résolubles, Dunod, Paris, 1972.

[ 3 ] L. Corwin and F. Greenleaf, Direct integral decompositions and multiplicities for induced representations of nilpotent Lie groups, Trans. Amer. Math. Soc., 304 (1987), 549-583.

[4] H. Fujiwara, Représentations monomiales des groupes de Lie résolubles exponentiels, The Orbit Method in Representation Theory, Progress in Math., 82 (1990), Birkhauser, 6184.

[ 5 ] A. Kleppner and R. Lipsman, The Plancherel formula for group extensions, Ann. Sci. École Normal Sup., 5 (1972), 459-516.

[6] R. Lipsman, Orbit theory and representations of Lie group with co-compact radical, J. Math. Pures Appl., 60 (1982), 17-39.

[ 7 ] R. Lipsman, Orbital parameters for induced and restricted representations, Trans. Amer. Math. Soc., 313 (1989), 433-473.

[8] R. Lipsman, Induced representations of completely solvable Lie groups, Ann. Scuola Normale Sup. Pisa, 17 (1990), 127-164.

[9] R. Lipsman, Induced representation of exponential solvable Lie groups, unpublished.

[10] I. Shchepochkina, Representations of solvable Lie groups, Functional Anal. Appl., 11 (1977), 159-161.

\author{
Department of Mathematics \\ UNIVERSITY OF MARYLAND \\ College Park, MD 20742, U.S.A.
}

